\title{
Internal dynamics of the galaxy cluster Abell 545
}

\section{The ideal case where to study the simultaneous formation of a galaxy system and its brightest galaxy}

\author{
R. Barrena ${ }^{1,2}$, M. Girardi ${ }^{3,4}$, W. Boschin ${ }^{5}$, S. De Grandi ${ }^{6}$, D. Eckert ${ }^{7}$, and M. Rossetti ${ }^{7}$ \\ ${ }^{1}$ Instituto de Astrofísica de Canarias, C/Vía Láctea s/n, 38205 La Laguna (Tenerife), Canary Islands, Spain \\ e-mail: rbarrena@iac.es \\ 2 Departamento de Astrofísica, Universidad de La Laguna, Av. del Astrofísico Franciso Sánchez s/n, 38205 La Laguna (Tenerife), \\ Canary Islands, Spain \\ ${ }^{3}$ Dipartimento di Fisica dell’Università degli Studi di Trieste, Sezione di Astronomia, via Tiepolo 11, 34143 Trieste, Italy \\ 4 INAF - Osservatorio Astronomico di Trieste, via Tiepolo 11, 34143 Trieste, Italy \\ 5 Fundación Galileo Galilei - INAF, Rambla José Ana Fernández Perez 7, 38712 Breña Baja (La Palma), Canary Islands, Spain \\ 6 INAF - Osservatorio Astronomico di Brera, via E. Bianchi 46, 23807 Merate (LC), Italy \\ 7 INAF - IASF Milano, via E. Bassini 15, 20133 Milano, Italy
}

Received 22 November 2010 / Accepted 2 March 2011

\begin{abstract}
Context. The mechanisms giving rise to diffuse radio emission in galaxy clusters, and in particular their connection with cluster mergers, are still debated.

Aims. We seek to explore the internal dynamics of the cluster Abell 545, which has been shown to host a radio halo. Abell 545 is also peculiar for hosting in its center a very bright, red, diffuse intracluster light due to an old, presumably metal-rich stellar population, so bright to be named as "star pile".

Methods. Our analysis is mainly based on redshift data for 110 galaxies acquired at the Telescopio Nazionale Galileo. We identify 95 cluster members and analyze the cluster internal dynamics by combining galaxy velocities and positions. We also use both multiband photometric data acquired at the Isaac Newton Telescope and X-ray data from the XMM-Newton Science Archive.

Results. We estimate the cluster redshift, $\langle z\rangle=0.1580$, a large line-of-sight (LOS) velocity dispersion $\sigma_{V} \sim 1200 \mathrm{~km} \mathrm{~s}^{-1}$, and ICM temperature $k T_{\mathrm{X}} \sim 8 \mathrm{keV}$. Our optical and X-ray analyses detect substructures. Optical data reveal three main galaxy clumps (one at the center hosting the peak of X-ray emission; one at NNW, and one at NE); and possibly a fourth clump at South. There is not a dominant galaxy and the four brightest galaxies avoid the cluster core $-\gtrsim 0.4 h_{70}^{-1} \mathrm{Mpc}$ distant from the cluster center - and are $\gtrsim 1500 \mathrm{~km} \mathrm{~s}^{-1}$ far from the mean cluster velocity. Two of these brightest galaxies are located in the NNW and NE clumps. The analysis of the X-ray surface brightness distribution provides us evidence of a disturbed dynamical phase: the strong NNW-SSE elongation, a western excess, and a sharp discontinuity in the northern region which is the likely signature of a shock. Located in the star pile region there is the brightest galaxy of the cluster core (CBCG) and a very compact elliptical galaxy, likely a M32-like dwarf. We show that the star pile, which has a previously determined redshift, has a similar redshift to that of the CBCG. Both the star pile and the CBCG are at rest in the cluster rest frame. The elongation of the star pile and its relative position with respect to the CBCG indicate the same direction pointed out by the NE clump.

Conclusions. The emerging picture of Abell 545 is that of a massive, $M\left(R<1.6 h_{70}^{-1} \mathrm{Mpc}\right)=(1.1-1.8) \times 10^{15} h_{70}^{-1} M_{\odot}$, very complex cluster with merging occurring along two directions. Abell 545 gives another proof in the favor of the connection between cluster merger and extended, diffuse radio emission. The star pile, likely due to the process of a brightest galaxy forming in the cluster core, is related to the accretion along the NE direction. Abell 545 represents a textbook cluster where to study the simultaneous formation of a galaxy system and its brightest galaxy.
\end{abstract}

Key words. galaxies: clusters: individual: Abell 545 - galaxies: clusters: general - galaxies: kinematics and dynamics

\section{Introduction}

Merging processes constitute an essential ingredient of the evolution of galaxy clusters (see Feretti et al. 2002, for a review). An interesting aspect of these phenomena is the possible connection between cluster mergers and extended, diffuse radio sources: halos and relics. The synchrotron radio emission of these sources demonstrates the existence of large-scale cluster magnetic fields and of widespread relativistic particles. Cluster mergers have been proposed to provide the large amount of energy necessary for electron re-acceleration to relativistic energies and for magnetic field amplification (Tribble 1993; Feretti 1999, 2002; Sarazin 2002). Radio relics ("radio gischts" as referred to by
Kempner et al. 2004), which are polarized and elongated radio sources located in the cluster peripheral regions, seem to be directly associated with merger shocks (e.g., Ensslin et al. 1998; Roettiger et al. 1999; Ensslin \& Gopal-Krishna 2001; Hoeft et al. 2004). Radio halos are sources that permeate the cluster volume in a similar way to the X-ray emitting gas of the intracluster medium (hereafter ICM) and are usually found unpolarized (but see Govoni et al. 2005). Radio halos are more likely to be associated with the turbulence following a cluster merger although the precise radio formation scenario remains unclear (re-acceleration vs. hadronic models e.g., Brunetti et al. 2009; Ensslin et al. 2011). Very recently, a unified halo-relic model has been presented in the framework of hadronic models where 
the time-dependence of the magnetic fields and of the cosmic ray distributions is taken into account to explain both halos and (most) relics (Keshet 2010). In this model the ICM magnetization is triggered by a merger event, in part but probably not exclusively in the wake of merger shocks.

The study of galaxy clusters with radio emissions offers a unique tool to estimate strength and structure of large-scale magnetic fields and might have important cosmological implications (see Dolag et al. 2004; and Ferrari et al. 2008, for recent reviews). If it will be confirmed and understood the connection between radio halos/relics and cluster mergers, one could use radio diffuse sources to directly follow the formation of the large scale structure. The study of clusters with radio halos/relics will likely contribute to quantify the effect of the non-thermal pressure to the estimate of mass and temperature in galaxy clusters (e.g., Loeb \& Mao 1994; Dolag \& Schindler 2000; Markevitch 2010) and, more in general, the thermal and non thermal effects of cluster mergers on global properties and cosmological parameters (e.g., Sarazin 2004).

Unfortunately, one has been able to study these phenomena only recently on the basis of a sufficient statistics, i.e. few dozen clusters hosting diffuse radio sources up to $z \sim 0.4$ (e.g., Giovannini et al. 1999; see also Giovannini \& Feretti 2002; Feretti 2005; Giovannini et al. 2009) with MACS J0717.5+3745 at $z=0.55$ being the most distant one (Bonafede et al. 2009). This allowed a few authors to attempt a classification (e.g., Kempner et al. 2004; Ferrari et al. 2008). It is expected that new radio telescopes will largely increase the statistics of diffuse sources (e.g., LOFAR, Cassano et al. 2010a) and allow the study of diffuse radio emission in low X-ray luminosity clusters to discriminate among theories of halo formation (e.g., Cassano et al. 2005; Ensslin et al. 2011).

From the observational point of view, there is growing evidence of the connection between diffuse radio emission and cluster mergers, since up to now diffuse radio sources have been detected only in merging systems (see, e.g., Cassano et al. 2010b). In most cases the cluster dynamical state has been derived from X-ray observations (see Buote 2002; Cassano et al. 2010b). X-ray studies of merging clusters are useful to discriminate between pre- and post-collision phases and to give useful information about the behavior of the ICM, in particular in the case of the detection of a shock. In fact, one can compute the shock Mach number from X-ray data, infer the shock wave velocity and the age of the merger, compare the dissipated energy with that required by the radio source (e.g., Sarazin 2002; Markevitch 2002; Finoguenov et al. 2010). This contributes to constrain the magnetic field and/or discriminate between models (e.g., Finoguenov et al. 2010).

Optical data are a powerful way to investigate the presence and the dynamics of cluster mergers, too (e.g., Girardi \& Biviano 2002). The spatial and kinematical analysis of member galaxies allow us to reveal and measure the amount of substructure, and to detect and analyze possible pre-merging clumps or merger remnants. This optical information is really complementary to $\mathrm{X}$-ray information since galaxies and the intracluster medium react on different timescales during a merger (see, e.g., numerical simulations by Roettiger et al. 1997). In this context, we are conducting an intensive observational and data analysis program to study the internal dynamics of clusters with diffuse radio emission by using member galaxies (DARC - Dynamical Analysis of Radio Clusters - project, see Girardi et al. 2007¹).

\footnotetext{
1 See also the web site of the DARC project http://adlibitum. oat.ts.astro.it/girardi/darc
}

The analysis based on the position/kinematics of member galaxies has the advantage that galaxies and dark matter (DM, i.e. the $70-80 \%$ of cluster mass) have similar spatial distributions in both quiet and substructured clusters as show by weak gravitational lensing data (e.g., Kneib et al. 1993; Okabe \& Umetsu 2008), in agreement with the fact that both galaxies and DM can be considered non collisional cluster components. The interesting consequence is that one can directly estimate (projected) positions velocities, and masses of subclusters using galaxies as tracer particles. Combined X-ray and optical information is exploited at their best in ad hoc numerical simulations of merging clusters where dark matter, gas and shock behaviors can be analyzed in function of the merger age (e.g., Springel \& Farrar 2007; Mastropietro \& Burkert 2008). However, for clusters formed by two main subclusters, one can use the optical observational information in the approximation of the analytical two-body model (Beers et al. 1982). The angle of view of the merger and the merger age are the two unknown quantities for which additional information can be used. This additional information is, e.g., the angle of view suggested by the relative efficiency of the substructure methods (Pinkney et al. 1996); the time elapsed since the subcluster collision as suggested by the X-ray temperature variation during the merger (Ricker \& Sarazin 2001) or as inferred from the shock Mach number derived from X-ray or radio data (see, e.g., Boschin et al. 2010; Girardi et al. 2010, for application of the bimodal model in DARC clusters).

As for the DARC project, we found evidence of major optical substructure for all the twelve clusters already analyzed on the base of enough statistics (60-170 members). These twelve clusters span a wide range of optical morphologies (showing two, three, many important clumps), view angles, and radio morphologies (double relics, relics, halos). This is the main reason why we are studying several individual clusters in order to face on a global classification and present a meaningful, statistical study. Most clusters exhibiting diffuse radio emission have a relatively high gravitational mass (higher than $0.7 \times 10^{15}$ within $2 h_{70}^{-1} \mathrm{Mpc}$; see Giovannini \& Feretti 2002) and, indeed, most DARC clusters are very massive clusters with few exceptions (Boschin et al. 2008).

A comprehensive statistical analysis of the whole DARC sample and the conclusions derived on the origin of halos/relics will be the subject of a future study. Here we focalize our study on the internal dynamics of Abell 545. In particular, we show as this cluster can be used to check models of the origin of halos/relics, although we stress that only the analysis on a large sample is expected to be really advantageous.

\subsection{Abell 545}

During our observational program, we have conducted an intensive study of the cluster Abell 545 (hereafter A545). A545 is a very rich, X-ray luminous cluster: Abell richness class $=4$ (the second richest cluster in the Abell catalog, Abell et al. 1989); $L_{\mathrm{X}}(0.1-2.4 \mathrm{keV})=5.67 \times 10^{44} h_{70}^{-2} \mathrm{Mpc} \mathrm{erg} \mathrm{s}^{-1}$ (Böhringer et al. 2004) and $k T_{\mathrm{X}}=5.5_{-2.1}^{+5.5} \mathrm{keV}$ (David et al. 1993).

Optically, the cluster is classified as Bautz-Morgan class III (Abell et al. 1989) and has a Rood-Sastry morphological type "I ${ }_{c}$ ", i.e. clumpy irregular (Struble \& Ftaclas 1994). According to the spectra for two luminous galaxies A545 lies at $z=0.152$ (Schneider et al. 1983).

A545 is peculiar for hosting a very bright diffuse light, so bright to be named as "star pile" by Struble (1988) who discovered a red, elongated low surface density feature located close 
to the cluster center of A545 on both the E and O plates of the Palomar Observatory Sky Survey. Struble interpreted it as intracluster matter. Based on archival VLT-images and long-slit spectra obtained with Gemini-GMOS, Salinas et al. (2007, hereafter S07) found that the star pile is indeed associated with A545, its velocity being $\sim 1300 \mathrm{~km} \mathrm{~s}^{-1}$ higher than that of a faint central galaxy. The spectra indicate an old, presumably metal-rich population. Its brightness profile is much shallower than that of typical cD-galaxies. However, as recognized by S07, the formation of this star pile remains elusive, until a dynamical analysis of the cluster itself become available.

Evidence for an unrelaxed state of A545 comes from X-ray data analyses. The X-ray structure of this cluster is elongated as reported by Buote \& Tsai (1996, from ROSAT data) who selected A545 as "reference" cluster for elliptical morphology. In particular, they found that A545 is the only cluster in their sample of 59 clusters that is highly elongated but does not display any obvious center offset, i.e., a center displacement from the outer emission (Jones \& Forman 1992). An image from the XMM archive also shows that A545 is strongly NNW-SSE elongated (see Fig. 6 of S07 and our analysis in Sect. 4). Its unrelaxed state is consistent with the absence of a cooling flow (White et al. 1997, from Einstein data). To date, the possibly involved subclusters have not been yet detected and there is no information about the merger age. Indeed, the internal dynamical of A545 has never been analyzed.

The presence of a giant radio halo in A545 was suggested by Giovannini et al. (1999) and then the halo was studied by Bacchi et al. (2003). The halo structure is centrally located and rather regular (slightly elongated in the NE-SW direction). The radio power of A545 accommodates well on the relation between radio power and dipole power ratio (Buote 2001) and on the relation between radio power and X-ray luminosity (Bacchi et al. 2003).

We included this cluster in our DARC sample and obtained new spectroscopic and photometric data from the Telescopio Nazionale Galileo (TNG) and the Isaac Newton Telescope (INT), respectively. Our present analysis is based on these optical data and XMM-Newton Science Archive data, too.

This paper is organized as follows. We present our new optical data and the cluster catalog in Sect. 2. We present our results about the cluster structure based on optical and X-ray data in Sects. 3 and 4, respectively. We discuss our results and present our conclusions in Sect. 5.

Unless otherwise stated, we indicate errors at the $68 \%$ confidence level (hereafter c.l.). Throughout this paper, we use $H_{0}=70 \mathrm{~km} \mathrm{~s}^{-1} \mathrm{Mpc}^{-1}$ and $h_{70}=H_{0} /\left(70 \mathrm{~km} \mathrm{~s}^{-1} \mathrm{Mpc}^{-1}\right)$ in a flat cosmology with $\Omega_{0}=0.3$ and $\Omega_{\Lambda}=0.7$. In the adopted cosmology, 1' corresponds to $\sim 164 h_{70}^{-1} \mathrm{kpc}$ at the cluster redshift.

\section{New data and galaxy catalog}

Multi-object spectroscopic observations of A545 were carried out at the TNG telescope in October 2009. We used DOLORES/MOS with the LR-B Grism 1, yielding a dispersion of $187 \AA / \mathrm{mm}$. We used the new $2048 \times 2048$ pixels E2V $\mathrm{CCD}$, with a pixel size of $13.5 \mu \mathrm{m}$. In total, we observed $4 \mathrm{MOS}$ masks for a total of 142 slits. We acquired three exposures of $1200 \mathrm{~s}$ for each mask. Wavelength calibration was performed using helium and neon-mercury lamps. Reduction of spectroscopic data was carried out using the IRAF ${ }^{2}$ package. Radial velocities were determined using the cross-correlation technique

\footnotetext{
${ }^{2}$ IRAF is distributed by the National Optical Astronomy Observatories, which are operated by the Association of Universities
}

(Tonry \& Davis 1979) implemented in the RVSAO package (developed at the Smithsonian Astrophysical Observatory Telescope Data Center). Each spectrum was correlated against five templates for a variety of galaxy spectral types: E, S0, Sa, $\mathrm{Sb}$, and Sc (Kennicutt 1992). The template producing the highest value of $\mathcal{R}$, i.e., the parameter given by RVSAO and related to the signal-to-noise ratio of the correlation peak, was chosen. Moreover, all spectra and their best correlation functions were examined visually to verify the redshift determination. In seven cases (IDs. 3, 31, 34, 42, 71, 72, and 77; see Table 1), we assumed the EMSAO redshift to be a reliable estimate of the redshift. A545.

Our spectroscopic catalog lists 110 galaxies in the field of

The nominal errors as given by the cross-correlation are known to be smaller than the true errors (e.g., Malumuth et al. 1992; Bardelli et al. 1994; Ellingson \& Yee 1994; Quintana et al. 2000). Duplicate observations for the same galaxy allowed us to estimate the true intrinsic errors in data of the same quality taken with the same instrument (e.g. Barrena et al. 2007a,b). Here we have double determinations for eleven galaxies, thus we decided to apply the correction that had already been applied in above studies. Hereafter we assume that true errors are larger than nominal cross-correlation errors by a factor of 1.3. For the eleven galaxies with two redshift estimates, we used the weighted mean of the two measurements and the corresponding errors. As for the radial velocities estimated through EMSAO we assumed the largest between the nominal error and $100 \mathrm{~km} \mathrm{~s}^{-1}$. As for the whole catalog, the median error in $c z$ is $52 \mathrm{~km} \mathrm{~s}^{-1}$.

Our photometric observations were carried out in with the Wide Field Camera (WFC), mounted at the prime focus of the $2.5 \mathrm{~m}$ INT telescope. We observed A545 in $g^{\prime}, r^{\prime}$ and $i^{\prime}$ SloanGunn filters in photometric conditions and a seeing of $\sim 1.5^{\prime \prime}$.

The WFC consists of a four-CCD mosaic covering a $33^{\prime} \times 33^{\prime}$ field of view, with only a $20 \%$ marginally vignetted area. We took eight exposures of $600 \mathrm{~s}$ in $g^{\prime}$ filter, nine frames of $400 \mathrm{~s}$ exposure in $r^{\prime}$ filter and eight exposures more of $400 \mathrm{~s}$ in $i^{\prime}$ filter. So a total of $4800 \mathrm{~s}$ in $g^{\prime}$ filter, and $3600 \mathrm{~s}$ in $r^{\prime}$ and $i^{\prime}$ bands. The observations were developed making a dithering pattern. This observing procedure allowed us to build a "supersky" frame that was used to correct our images for fringing patterns (Gullixson 1992). In addition, the dithering helped us to clean cosmic rays and avoid the effects of gaps between the CCDs in the final images. Another problem associated with the wide field frames is the distortion of the field. To match the photometry of several filters, a good astrometric solution is needed to take into account these distortions. Using the imcoords IRAF tasks and taking as a reference the USNO B1.0 catalog, we were able to find an accurate astrometric solution (rms $\sim 0.4^{\prime \prime}$ ) across the full frame. The photometric calibration was performed by observing standard Landolt fields (Landolt 1992) calibrated in the SloanGunn system.

We finally identified galaxies in our $g^{\prime}, r^{\prime}$ and $i^{\prime}$ images and measured their magnitudes with the SExtractor package (Bertin \& Arnouts 1996) and AUTOMAG procedure. Objects were identified as imposing that they cover a certain minimum area and have a number counts above a limiting threshold taking the sky local background as a reference. The limiting size and flux were 16 pixels and 1.5 standard deviation of the sky counts, respectively. The selected limiting size corresponds to an apparent size of $1.3^{\prime \prime}$, which is about the minimum seeing size during

for Research in Astronomy, Inc., under cooperative agreement with the National Science Foundation. 
Table 1. Velocity catalog of 110 spectroscopically measured galaxies in the field of the cluster A545.

\begin{tabular}{|c|c|c|c|c|c|c|c|}
\hline ID & $\begin{array}{c}\alpha(\mathrm{J} 2000), \delta(\mathrm{J} 2000) \\
\mathrm{h}: \mathrm{m}: \mathrm{s},{ }^{\circ}:^{\prime}::^{\prime \prime}\end{array}$ & $g^{\prime}$ & $r^{\prime}$ & $i^{\prime}$ & $\begin{array}{l}v \\
(\mathrm{kms}\end{array}$ & $\begin{array}{c}\Delta v \\
\left.s^{-1}\right)\end{array}$ & Mem. \\
\hline 1 & $053209.06,-113052.4$ & 18.72 & 17.82 & 17.53 & 46877 & 33 & 1 \\
\hline 2 & $053210.89,-113305.9$ & 17.05 & 16.13 & 15.89 & 48992 & 35 & 1 \\
\hline 3 & $053211.41,-113108.3$ & 19.92 & 19.22 & 19.01 & 103532 & 100 & 0 \\
\hline 4 & $053211.93,-113259.8$ & 18.64 & 17.95 & 17.60 & 47906 & 35 & 1 \\
\hline 5 & 0532 13.27, - 113325.4 & 19.61 & 18.70 & 18.37 & 45015 & 39 & 1 \\
\hline 6 & $053213.47,-113048.6$ & 18.05 & 17.33 & 17.04 & 45364 & 40 & 1 \\
\hline 7 & $053213.93,-113121.8$ & 19.30 & 18.59 & 18.33 & 46967 & 38 & 1 \\
\hline 8 & $053214.13,-113426.5$ & 20.61 & 19.35 & 19.03 & 72394 & 55 & 0 \\
\hline 9 & $053214.98,-113243.6$ & 18.14 & 17.50 & 17.24 & 44558 & 34 & 1 \\
\hline 10 & 0532 & & & 18.23 & 47521 & 56 & 1 \\
\hline 11 & $053216.11,-11340$ & 20.22 & 19.29 & 18.92 & 47956 & 72 & 1 \\
\hline 12 & $053216.64,-113442$ & 20.74 & 19.46 & 19.01 & 81561 & 45 & 0 \\
\hline 13 & $053216.75,-112946.7$ & 20.31 & 19.44 & 19.06 & 47925 & 64 & 1 \\
\hline 14 & $053216.88,-113453.3$ & 19.40 & 18.71 & 18.40 & 46953 & 42 & 1 \\
\hline 15 & 0532 17.37, -1129 18.7 & 19.29 & 18.43 & 18.13 & 47329 & 56 & 1 \\
\hline 16 & $053217.64,-113339.4$ & 18.70 & 17.77 & 17.41 & 48957 & 35 & 1 \\
\hline 17 & $053217.79,-113151.1$ & 20.99 & 20.14 & 19.81 & 48992 & 59 & 1 \\
\hline 18 & $053218.01,-113356.6$ & 20.28 & 19.46 & 19.12 & 47164 & 64 & 1 \\
\hline 19 & $053218.08,-112900.7$ & 19.91 & 18.99 & 18.62 & 76942 & 62 & 0 \\
\hline 20 & $053218.21,-113440.5$ & 18.21 & 17.26 & 16.88 & 47765 & 42 & 1 \\
\hline 21 & $053218.33,-113100.6$ & 20.26 & 19.45 & 19.12 & 48803 & 60 & 1 \\
\hline 22 & $053218.88,-113012$ & 19.52 & 18.63 & 18.27 & 46794 & 43 & 1 \\
\hline 23 & 05321 & 20.05 & 19.22 & 18.90 & 47230 & 64 & 1 \\
\hline 24 & 0532 & 17.92 & 17.31 & 17.02 & 48594 & 72 & 1 \\
\hline 25 & 0532 & 19. & 18. & 18.45 & 46621 & 40 & 1 \\
\hline 26 & $053219.37,-$ & 20.66 & 19.68 & 19.35 & 49423 & 107 & 1 \\
\hline 27 & $053219.45,-112934.8$ & 17.70 & 16.73 & 16.36 & 49449 & 38 & 1 \\
\hline 28 & $053219.45,-113534.0$ & 19.01 & 18.21 & 17.79 & 46433 & 48 & 1 \\
\hline 29 & $053220.00,-113502.0$ & 18.12 & 17.41 & 17.11 & 45708 & 40 & 1 \\
\hline 30 & $053220.18,-113315.5$ & 20.16 & 19.20 & 18.80 & 46677 & 59 & 1 \\
\hline 31 & $053220.63,-113058.2$ & 19.12 & 18.50 & 18.20 & 68252 & 116 & 0 \\
\hline 32 & $053220.83,-113410.0$ & 20.53 & 19.65 & 19.25 & 47072 & 73 & 1 \\
\hline 33 & $053221.52,-113258.5$ & 20.34 & 19.45 & 19.09 & 45745 & 88 & 1 \\
\hline 34 & $053221.56,-112815.6$ & 18.54 & 17.73 & 17.35 & 67715 & 100 & 0 \\
\hline 35 & $053221.56,-112923.0$ & 19.30 & 18.64 & 18.37 & 50360 & 44 & 1 \\
\hline 36 & $053221.83,-113$ & 18.90 & 17.98 & 17.60 & 48049 & 25 & 1 \\
\hline 37 & $053221.84,-113224.0$ & 18.91 & 17.96 & 17.57 & 47452 & 29 & 1 \\
\hline 38 & 0532 21.94, & & 18.76 & 18.34 & 45527 & 73 & 1 \\
\hline 39 & 0 & 9.51 & 18.68 & 18.32 & 46272 & 49 & 1 \\
\hline 40 & $053221.99,-113233.2$ & 18.56 & 17.82 & 17.52 & 45652 & 22 & 1 \\
\hline 41 & $053222.14,-113538.2$ & 20.52 & 19.99 & 19.91 & 50629 & 61 & 1 \\
\hline 42 & $053222.36,-113050.6$ & 20.46 & 19.93 & 19.59 & 116937 & 100 & 0 \\
\hline 43 & $053222.58,-112815.2$ & 19.64 & 18.45 & 17.98 & 67832 & 73 & 0 \\
\hline 44 & $053223.06,-112955.7$ & 20.03 & 19.05 & 18.68 & 49512 & 52 & 1 \\
\hline 45 & $053223.46,-113446.8$ & 19.10 & 18.18 & 17.82 & 47744 & 44 & 1 \\
\hline 46 & $053223.49,-112936.6$ & 20.29 & 19.39 & 19.02 & 46560 & 52 & 1 \\
\hline 47 & $053223.55,-113154.8$ & 18.73 & 17.97 & 17.53 & 41964 & 51 & 0 \\
\hline 48 & $053223.77,-113246.0$ & 18.22 & 17.27 & 16.89 & 46105 & 22 & 1 \\
\hline 49 & $053223.82,-112925.8$ & 18.61 & 17.73 & 17.31 & 44975 & 38 & 1 \\
\hline 50 & $053224.14,-113232.6$ & 19.02 & 18.05 & 17.63 & 48736 & 42 & 1 \\
\hline 51 & $053224.18,-113420.0$ & 19.01 & 18.12 & 17.74 & 47044 & 42 & 1 \\
\hline 52 & 0532 24.30, & 1.77 & 20.64 & 20.33 & 46653 & 109 & 1 \\
\hline 53 & $41,-113$ & 19.51 & 18.75 & 18.51 & 48216 & 66 & 1 \\
\hline 54 & $053224.46,-113302.8$ & 19.57 & 18.55 & 18.46 & 48636 & 40 & 1 \\
\hline 55 & $053224.60,-113230.4$ & 19.26 & 18.32 & 17.86 & 47212 & 35 & 1 \\
\hline 56 & $053224.73,-113258.3$ & 19.58 & 18.53 & 18.27 & 48907 & 74 & 1 \\
\hline 57 & $053224.90,-113238.7$ & 20.88 & 19.97 & 19.54 & 46354 & 109 & 1 \\
\hline 58 & $053224.93,-113555.6$ & 21.02 & 19.34 & 18.80 & 117147 & 90 & 0 \\
\hline 59 & $053225.20,-113032.7$ & 20.30 & 19.45 & 19.11 & 46321 & 153 & 1 \\
\hline 60 & $053225.28,-113002.0$ & 18.54 & 17.60 & 17.18 & 47505 & 35 & 1 \\
\hline 61 & $053225.33,-113500.0$ & 18.74 & 17.91 & 17.59 & 48896 & 51 & 1 \\
\hline 62 & $053225.39,-112854.4$ & 19.31 & 18.52 & 18.09 & 49441 & 53 & 1 \\
\hline 63 & $053225.40,-113206.2$ & 20.20 & 19.35 & 18.96 & 45711 & 87 & 1 \\
\hline 64 & $053225.76,-113234.2$ & 18.31 & 17.29 & 16.71 & 47071 & 26 & 1 \\
\hline
\end{tabular}

Table 1. continued.

\begin{tabular}{cccccccc}
\hline \hline ID & $\begin{array}{c}\alpha(\mathrm{J} 2000), \delta(\mathrm{J} 2000) \\
\mathrm{h}: \mathrm{m}: \mathrm{s},{ }^{\circ}:^{\prime}:^{\prime \prime}\end{array}$ & $g^{\prime}$ & $r^{\prime}$ & $i^{\prime}$ & $\begin{array}{l}v \\
\left(\mathrm{kms}^{-1}\right)\end{array}$ \\
\hline
\end{tabular}

$65 \quad 0532 \quad 25.96,-113359.419 .46 \quad 18.55 \quad 18.1746995 \quad 36$

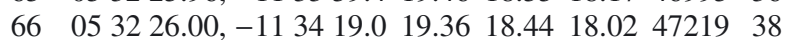

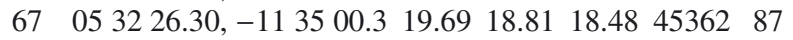

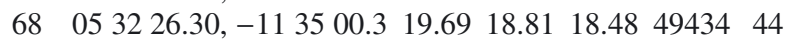
$69053226.43,-113237.7 \quad 18.8917 .92 \quad 17.4146297 \quad 35$

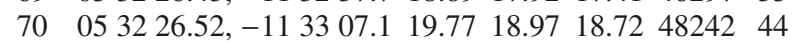

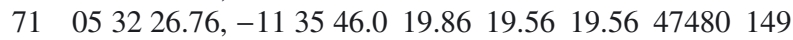

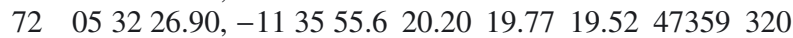

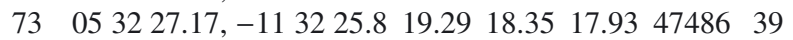

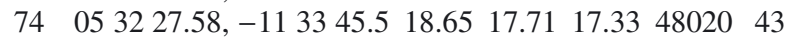

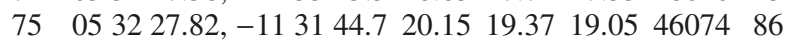

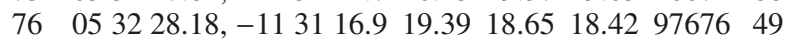

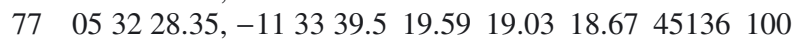
$78 \quad 053228.46,-113033.619 .94 \quad 19.01 \quad 18.63 \quad 45406 \quad 51$

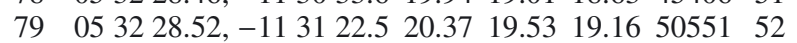

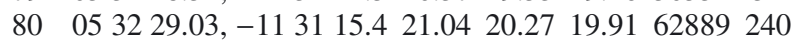

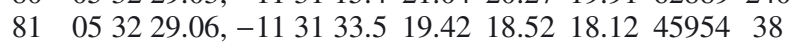

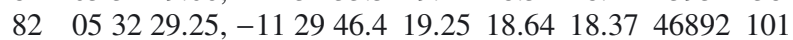

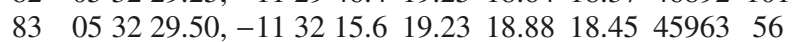
$84 \quad 0532 \quad 29.55,-113142.120 .46 \quad 19.70 \begin{array}{lllllll}19.47421 & 52\end{array}$

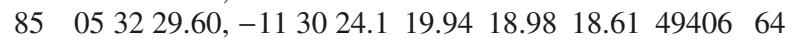
$86 \quad 0532 \quad 29.75,-113517.420 .18 \quad 18.75 \quad 18.20 \quad 97303 \quad 35$

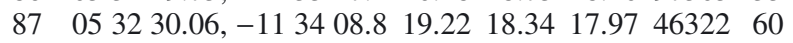
$88 \quad 0532 \quad 30.14,-113515.620 .71 \quad 19.41 \quad 18.8197292 \quad 70$

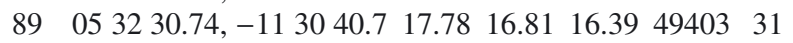
$90 \quad 053231.09,-113142.1 \quad 18.83 \quad 18.40 \quad 18.174622964$

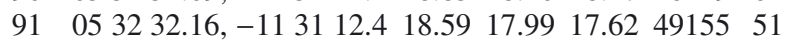

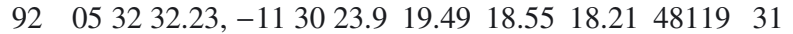

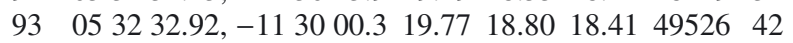
$94 \quad 0532 \quad 33.50,-113516.419 .59 \quad 18.71 \quad 18.21 \quad 47562 \quad 51$

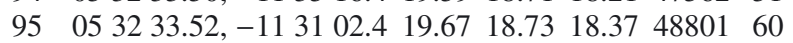

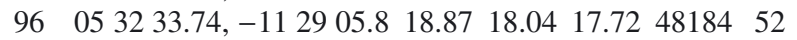

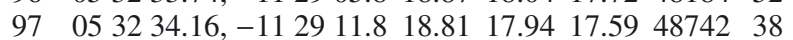

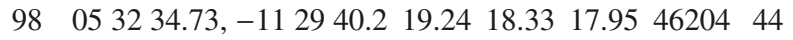

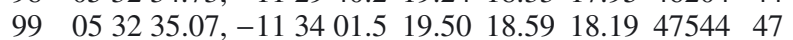
$100053235.65,-113414.5 \quad 18.45 \quad 17.54 \quad 17.1847375 \quad 34$

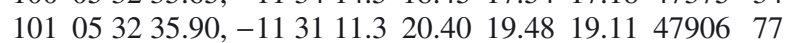

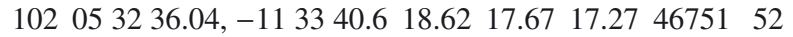

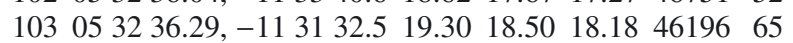

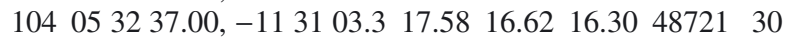

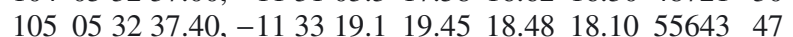
$106053237.63,-113248.2 \quad 19.39 \quad 18.56 \quad 18.2346266 \quad 34$

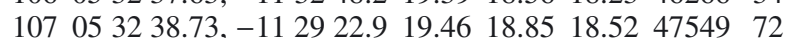
$108053239.41,-113317.520 .07 \quad 18.9818 .414579496$

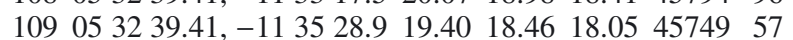

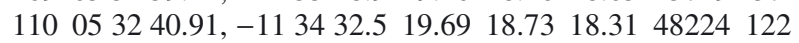

the observations. We have performed careful visual inspections of the frames in order to deal with the best combination of the above parameters that remove spurious objects from the cata$\log$.

In a few cases (e.g., close companion galaxies, galaxies close to defects of the CCD) the standard SExtractor photometric procedure failed. In these cases, we computed magnitudes by hand. This method consisted of assuming a galaxy profile of a typical elliptical galaxy and scaling it to the maximum observed value. The integration of this profile provided an estimate of the magnitude. This method is similar to PSF photometry, but assumes a galaxy profile, which is more appropriate in this case.

As a final step, we estimated and corrected the Galactic extinction $A_{g^{\prime}} \sim 0.65, A_{r^{\prime}} \sim 0.45$ and $A_{i^{\prime}} \sim 0.35$ using Schlegel et al. (1998) reddening and extinction maps computed from IRAS and COBE/DIRBE data. We estimated that 
R. Barrena et al.: Internal dynamics of the galaxy cluster Abell 545

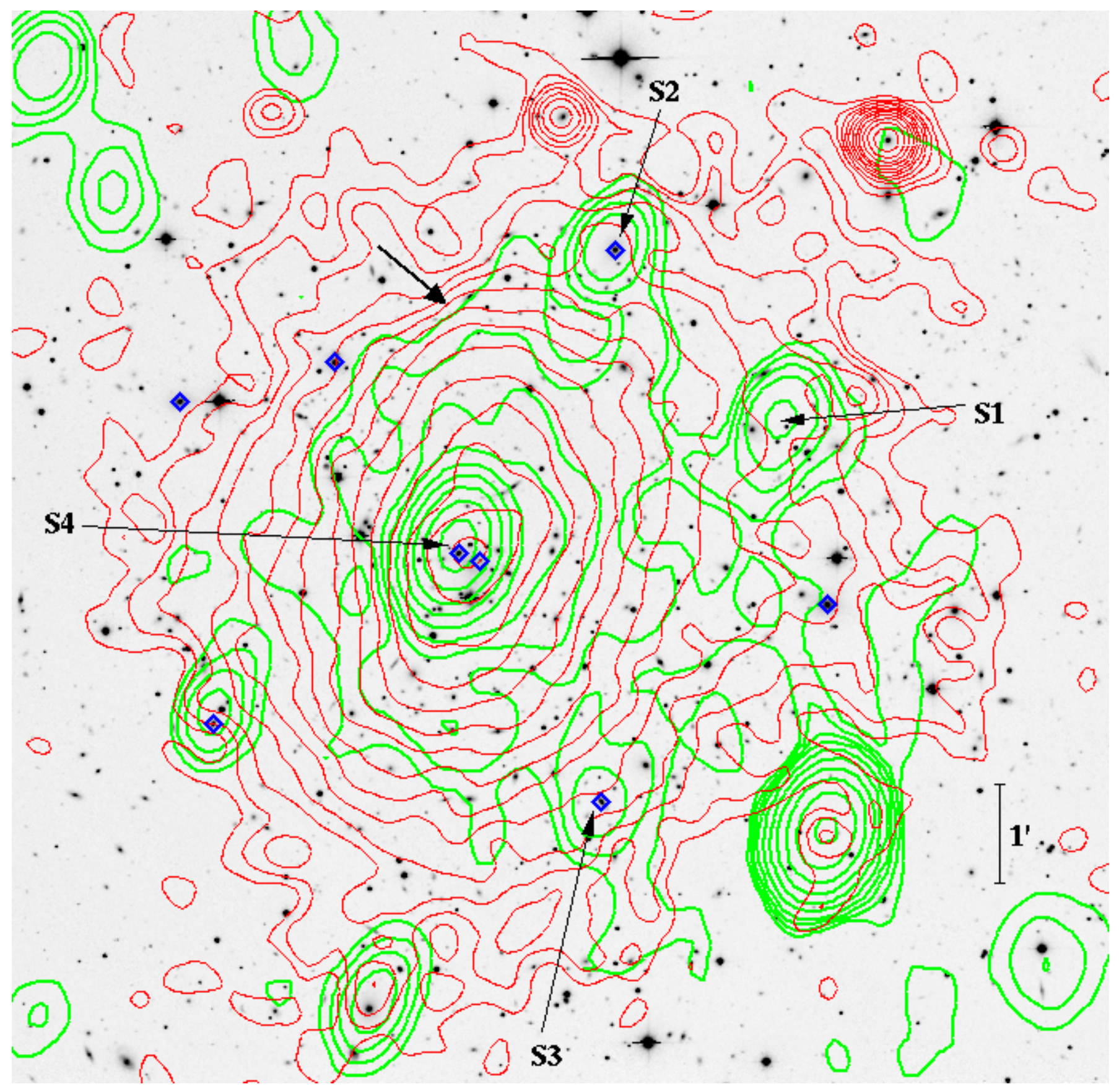

Fig. 1. INT $r^{\prime}$-band image of the cluster A545 (north at the top and east to the left) with, superimposed, the contour levels of the XMM archival image (ID. 0304750101) in the 0.4-2 keV energy range (thin/red contours) and the contour levels of a VLA radio image at $1.4 \mathrm{GHz}$ (thick/green contours, see Bacchi et al. 2003). Blue rotate squares highlight the positions of bright galaxies cited in the text. Labels S1, S2, S3 and S4 indicate the positions of radio sources listed by Bacchi et al. In particular, the galaxy indicated by "S4" is the brightest galaxy in the cluster central region (CBCG). In the northern region, an arrow highlights a sharp discontinuity in the X-ray surface brightness (see text).

our photometric sample is complete down to $g^{\prime}=23.6(24.5)$, $r^{\prime}=22.3(23.5)$ and $i^{\prime}=21.3(22.8)$ for $S / N=5(3)$ within the observed field.

We assigned magnitudes to all galaxies of our spectroscopic catalog. Table 1 lists the velocity catalog (see also Fig. 2): identification number of each galaxy, ID (Col. 1); right ascension and declination, $\alpha$ and $\delta$ (J2000, Col. 2); $g^{\prime}, r^{\prime}$, and $i^{\prime}$ magnitudes (Cols. 3-5); heliocentric radial velocity, $v=c z_{\odot}$ (Col. 6) with error, $\Delta \mathrm{v}$ (Col. 7); and code for membership (1/0 for members/nonmembers, respectively; Col. 8).

No evident dominant galaxy is present in the cluster, e.g. the brightest galaxy in our catalog (ID. 2) is only 0.5 mag brighter in the $r^{\prime}$ band then the second brightest galaxy (ID. 104) and both are far from the cluster center as indicated by the peak of the X-ray emission [RA $=05^{\mathrm{h}} 32^{\mathrm{m}} 25^{\mathrm{s}} .6, \mathrm{Dec}=-11^{\circ} 32^{\prime} 37^{\prime \prime}$ (J2000.0)] derived from our analysis of the XMM-Newton data (see Sect. 4). Other galaxies brighter than 17 mag in the $r^{\prime}$ band are IDs. 27 and 89 . Hereafter we refer to these galaxies as the BCG1, BCG2, BCG3, and BCG4. These galaxies are $\gtrsim 0.4 h_{70}^{-1} \mathrm{Mpc}$ distant from the cluster center. As for the cluster central region, the most luminous galaxy is ID. 64 (hereafter CBCG), which is located just close to the peak of the X-ray emission and the location of the star pile (see Figs. 1 and 2). For the CBCG we measure a redshift concordant with that of the 


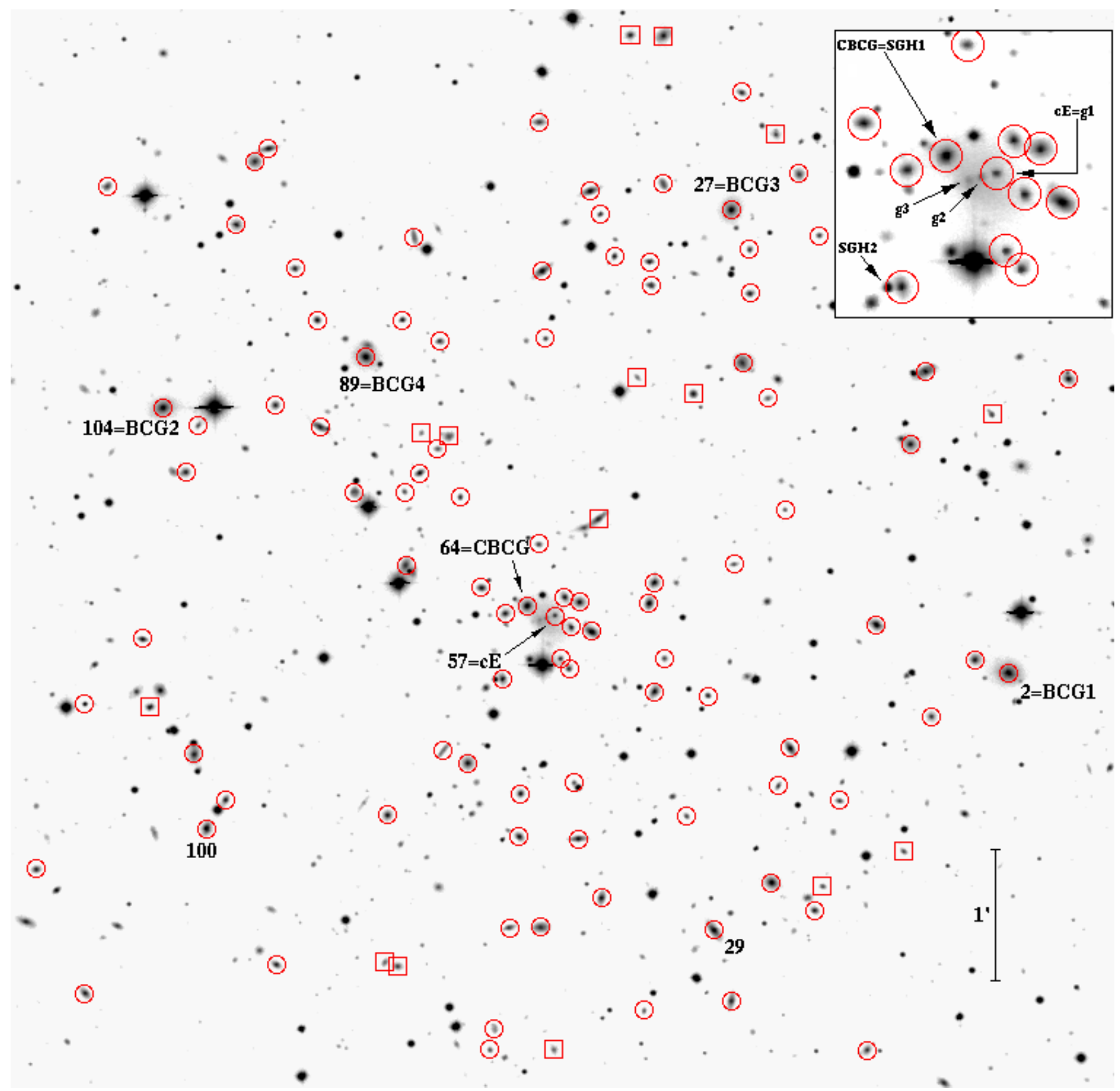

Fig. 2. INT $r^{\prime}$-band image of the cluster A545 (north at the top and east to the left). Circles and squares indicate spectroscopically cluster members and non-members, respectively (see Table 1). Labels indicate the IDs of cluster galaxies cited in the text. In the insect figure the corresponding IDs of S07 are given, too.

star pile (as obtained by S07 through Gemini-GMOS long-slit spectra).

Our spectroscopic catalog lists some radio galaxies observed in the field of A545. Figure 2 of Bacchi et al. (2003) show four discrete sources in the region of the radio halo: the optical counterparts of S2 and S3 are the cluster members IDs. 27 and 29, respectively. The radio source $\mathrm{S} 4$ is located in the crowed central cluster region and its most likely counterpart is the luminous $\mathrm{CBCG}$. As for $\mathrm{S} 1$ there is no an obvious counterpart. Figure 2 of Bacchi et al. also shows other three radio sources at the border/outside the radio halo: the one at SE has as possible counterpart the cluster member ID. 100; for the one at South we have not acquired the redshift of the counterpart; the one at SW has no counterpart in our optical image.
Another galaxy to be mentioned is ID. 57, which, according to S07, is a very compact object. We find that it is a cluster member thus discarding the possibility that it is a star projected onto the cluster field. Thus ID. 57 resembles the characteristics of a M32-like dwarf object of which only a few representatives are known (e.g., Chilingarian et al. 2007, 2009). Moreover, we find that its colors $\left(g^{\prime}-r^{\prime}=0.91, r^{\prime}-i^{\prime}=0.43\right)$ and spectrum resemble a typical early type galaxy. Hereafter we indicate this galaxy as "cE".

\section{Analysis of the optical data}

\subsection{Member selection}

To select cluster members among the 110 galaxies with redshifts, we follow a two-step procedure. We first perform the 1D 


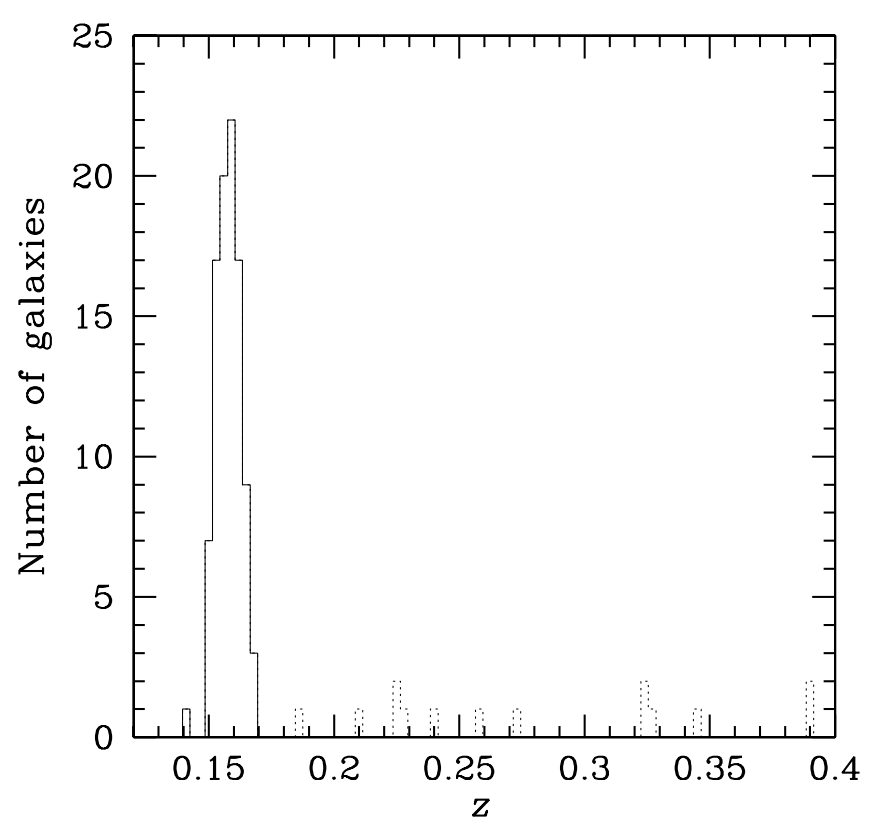

Fig. 3. Redshift galaxy distribution. The solid line histogram refers to the 96 galaxies assigned to A545 according to the DEDICA reconstruction method.

adaptive-kernel method (hereafter DEDICA, Pisani 1993, and 1996; see also Fadda et al. 1996; Girardi et al. 1996). We search for significant peaks in the velocity distribution at $>99 \%$ c.l. This procedure detects A545 as a peak at $z \sim 0.157$ populated by 96 galaxies considered as candidate cluster members (in the range $41964 \leq v \leq 50629 \mathrm{~km} \mathrm{~s}^{-1}$, see Fig. 3). The 14 non-members are background galaxies.

All the galaxies assigned to the cluster peak are analyzed in the second step, which uses the combination of position and velocity information, i.e., the "shifting gapper" method by Fadda et al. (1996). This procedure rejects galaxies that are too far in velocity from the main body of galaxies within a fixed bin that shifts along the distance from the cluster center. The procedure is iterated until the number of cluster members converges to a stable value. Following Fadda et al. (1996), we use a gap of $1000 \mathrm{~km} \mathrm{~s}^{-1}$ - in the cluster rest-frame - and a bin of $0.6 h_{70}^{-1} \mathrm{Mpc}$, or large enough to include 15 galaxies. As for the center of A545, we adopt the position of the peak of the $\mathrm{X}$-ray emission (see above). The "shifting gapper" procedure rejects another one obvious interloper very far from the main body $\left(>2000 \mathrm{~km} \mathrm{~s}^{-1}\right)$ but that survived the first step of our member selection procedure (see the red cross in Fig. 5). We obtain a sample of 95 fiducial members (see Figs. 4 and 5).

\subsection{Global cluster properties}

By applying the biweight estimator to the 95 cluster members (Beers et al. 1990, ROSTAT software), we compute a mean cluster redshift of $\langle z\rangle=0.1580 \pm 0.0004$, i.e. $\langle v\rangle=47373 \pm$ $125 \mathrm{~km} \mathrm{~s}^{-1}$. We estimate the LOS velocity dispersion, $\sigma_{V}$, by using the biweight estimator and applying the cosmological correction and the standard correction for velocity errors (Danese et al. 1980). We obtain $\sigma_{V}=1220_{-68}^{+82} \mathrm{~km} \mathrm{~s}^{-1}$, where errors are estimated through a bootstrap technique.

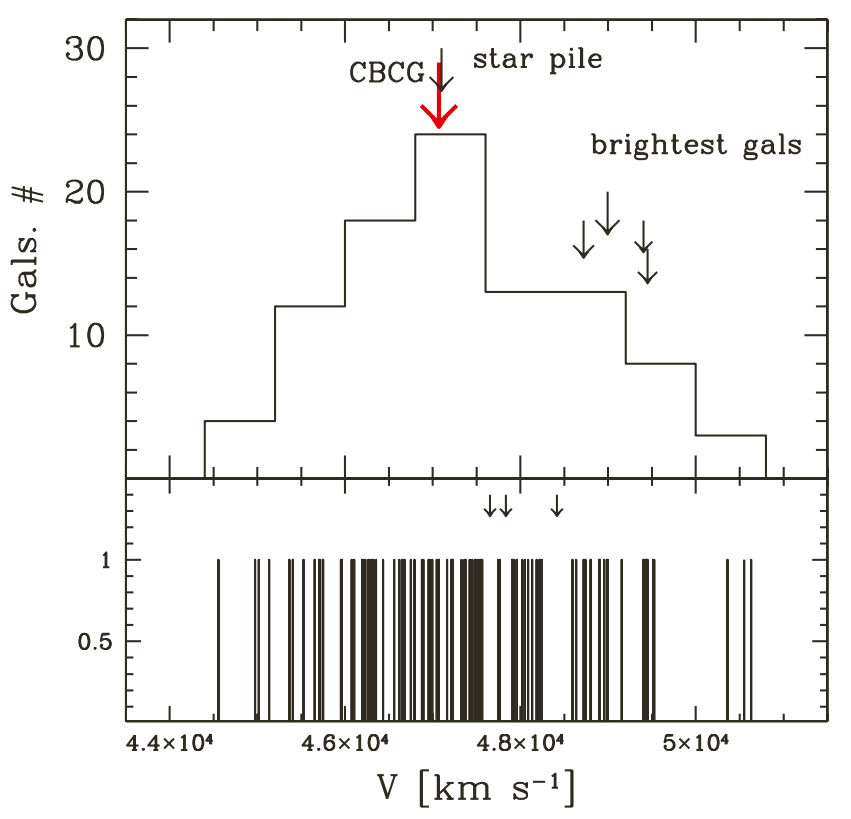

Fig. 4. The 95 galaxies assigned to the cluster. Upper panel: velocity distribution. The arrows indicate the velocities of the four brightest galaxies, the velocity of the central bright galaxy CBCG (bigger red arrow), and the velocity of the star pile. Lower panel: stripe density plot where the arrows indicate the positions of the significant gaps.

To evaluate the robustness of the $\sigma_{V}$ estimate, we analyze the velocity dispersion profile (Fig. 5). The integral profile rises out to $\sim 0.1 h_{70}^{-1} \mathrm{Mpc}$ and then flattens suggesting that a robust value of $\sigma_{V}$ is asymptotically reached in the external cluster regions, as found for most nearby clusters (e.g., Fadda et al. 1996; Girardi et al. 1996).

\subsection{Velocity distribution}

We analyze the velocity distribution to search for possible deviations from Gaussianity that might provide important signatures of complex dynamics. For the following tests, the null hypothesis is that the velocity distribution is a single Gaussian.

We estimate three shape estimators, i.e., the kurtosis, the skewness, and the scaled tail index (see, e.g., Bird \& Beers 1993). According to the value of the normalized kurtosis $(K U R T=0.627)$, and the scaled tail index $(S T I=0.873)$ the velocity distribution is marginally differing from a Gaussian at the $90-95 \%$ c.l. (see Table 2 of Bird \& Beers 1993).

We then investigate the presence of gaps in the velocity distribution. We follow the weighted gap analysis presented by Beers et al. (1991, 1992; ROSTAT software). We look for normalized gaps larger than 2.25 since in random draws of a Gaussian distribution they arise at most in about $3 \%$ of the cases, independent of the sample size (Wainer and Schacht 1978). We detect three significant gaps (at the $99.4 \%, 97 \%, 99.95 \%$ c.1.s), which divide the cluster into four groups of 58,2,11, and 24 galaxies from low to high velocities (hereafter GV1, GV2, GV3 and GV4, see Fig. 4). The CBCG is assigned to the GV1 group. The BCG1-2-3-4 are all assigned to the GV4 group.

Following Ashman et al. (1994), we also apply the Kaye's mixture model (KMM) algorithm. This test does not find that a two or a three or a four-groups partition would provide a 


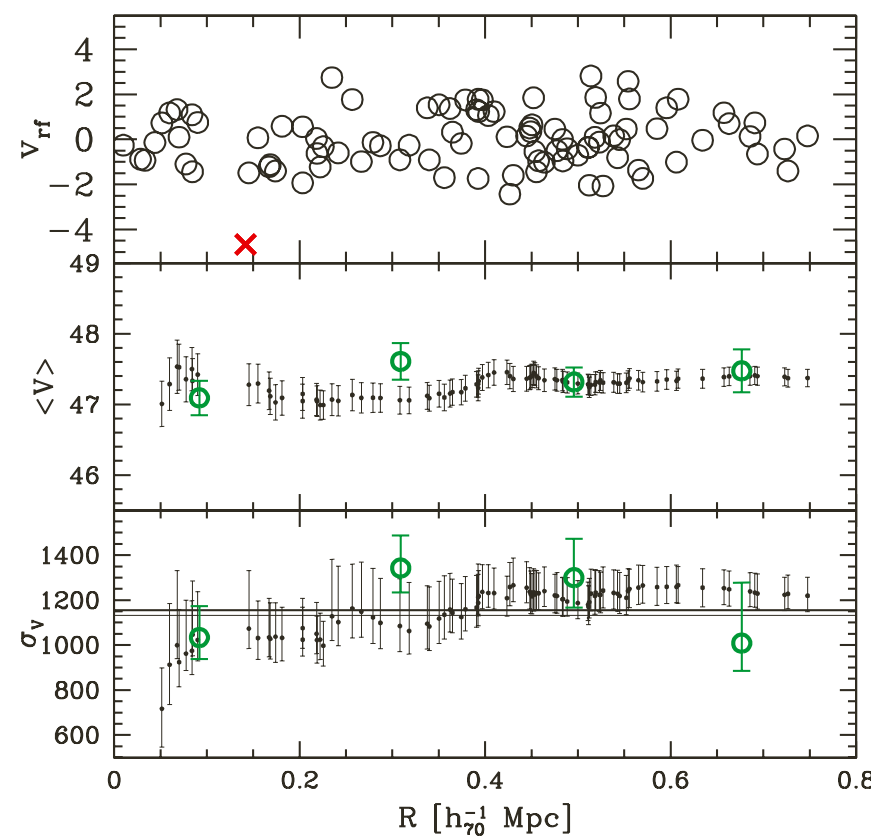

Fig. 5. Top panel: rest-frame velocity vs. projected distance from the cluster center of cluster members (circles). The red cross indicates the galaxy rejected by the "shifting gapper" selection procedure. Middle and bottom panels: differential (big circles) and integral (small points) profiles of mean velocity and LOS velocity dispersion, respectively. For the differential profiles, we plot the values for four annuli from the center of the cluster, each of $0.2 h_{70}^{-1} \mathrm{Mpc}$ (large green symbols). For the integral profiles, the mean and dispersion at a given (projected) radius from the cluster-center is estimated by considering all galaxies within that radius - the first value computed on the five galaxies closest to the center. The error bands at the $68 \%$ c.l. are also shown. In the lower panel, the horizontal line represents the X-ray temperature with the respective errors transformed in $\sigma_{V}$ assuming the density-energy equipartition between ICM and galaxies, i.e. $\beta_{\text {spec }}=1$ (see Sect. 5).

significantly more accurate description of the velocity distribution than a single Gaussian.

\subsection{Analysis of the $2 D$ galaxy distribution}

By applying the 2D adaptive-kernel method to the positions of A545 galaxy members, the most significant, dense peak is that coinciding with the peak of the X-ray emission ("C" label in Fig. 6). Other three significant peaks are shown: one located at North, slightly NNW); one located at NE; and one located at South with respect to the position of the main one. DEDICA assigns the CBCG and BCG1 to the $\mathrm{C}$ group, the BCG3 to the NNW group, and the BCG2 and BCG4 to the NE group. The CBCG, BCG3, and BCG4 are particularly close to their respective peaks $\left(0.05,0.11\right.$, and $0.16 h_{70}^{-1} \mathrm{Mpc}$, respectively, see Fig. 6). However, our spectroscopic data do not cover the entire cluster field and are affected by magnitude incompleteness. To overcome these problems, from our photometric catalog we use our photometric data sample which covers a larger spatial region.

In our photometric catalog we select likely members on the base of both $\left(r^{\prime}-i^{\prime}\right)$ and $\left(g^{\prime}-r^{\prime}\right)$ colors. Goto et al. (2002) showed that there is a small tilt in the color-magnitude relations $\left(r^{\prime}-i^{\prime}\right)$ vs. $r^{\prime}$ and $\left(g^{\prime}-r^{\prime}\right)$ vs. $r^{\prime}$ and that the scatter in the latter relation is roughly the double than the scatter in the first one $(0.040$

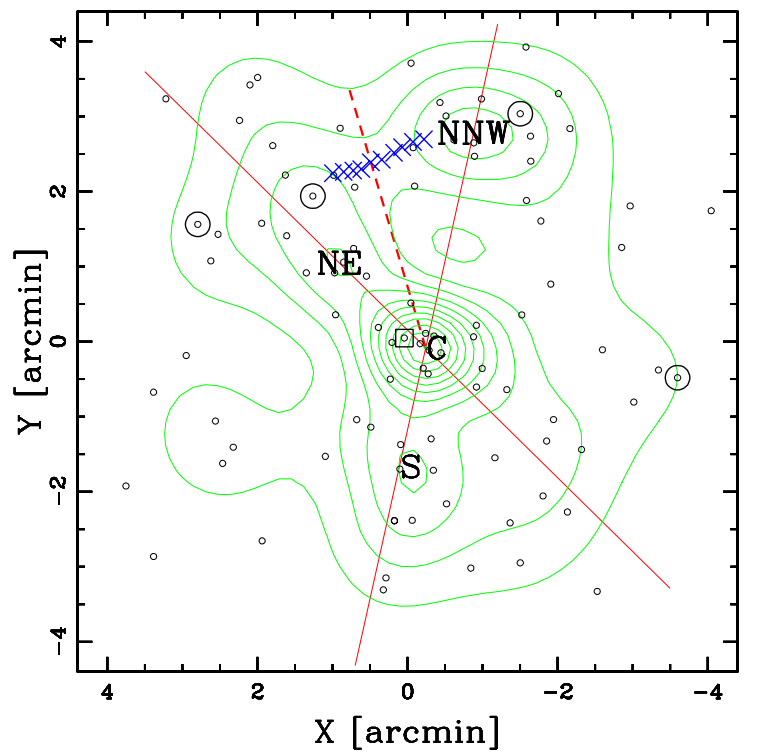

Fig. 6. Spatial distribution on the sky and relative isodensity contour map of spectroscopic cluster members, obtained with the 2D DEDICA method. The X-ray peak is taken as the cluster center. The location of the four brightest galaxies are indicated by large circles. The square indicates the central luminous galaxy CBCG. The four significant peaks are labelled. The two (red) solid slides and the dashed line indicate the two likely merging directions and their bisecting, respectively. Blue crosses indicate, in a schematic way, the sharp discontinuity detected in the X-ray surface brightness (see Sect. 4 for details).

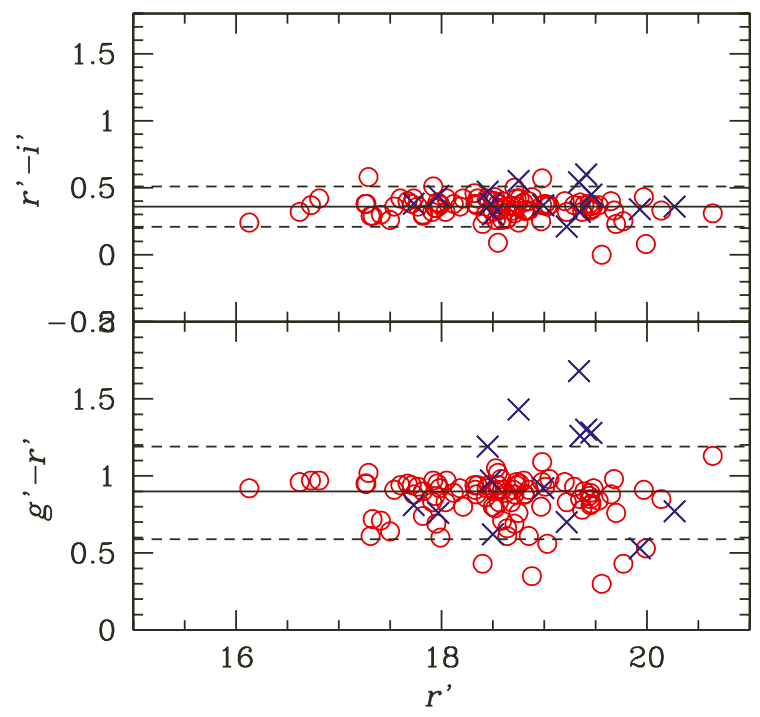

Fig. 7. Upper panel: $r^{\prime}-i^{\prime}$ vs. $r^{\prime}$ diagram for galaxies with available spectroscopy. Red circles and blue crosses indicate cluster members and non members. The solid line gives the median color determined on member galaxies; the dashed lines are drawn at \pm 0.15 mag from this value. Lower panel: $g^{\prime}-r^{\prime}$ vs. $r^{\prime}$ diagram for galaxies with available spectroscopy. The dashed lines are drawn at \pm 0.3 mag from the median value determined on member galaxies.

and $0.081 \mathrm{mag}$, respectively). Out of our photometric catalog we consider as "likely cluster members" those objects having $\left(r^{\prime}-i^{\prime}\right)$ and $\left(g^{\prime}-r^{\prime}\right)$ lying within \pm 0.15 and \pm 0.3 from the median values of $r^{\prime}-i^{\prime}=0.36$ and $g^{\prime}-r^{\prime}=0.89$ colors of the spectroscopically cluster members, i.e., we reject those objects having colors $\gtrsim 3$ sigma far from the red sequence (see Fig. 7). 


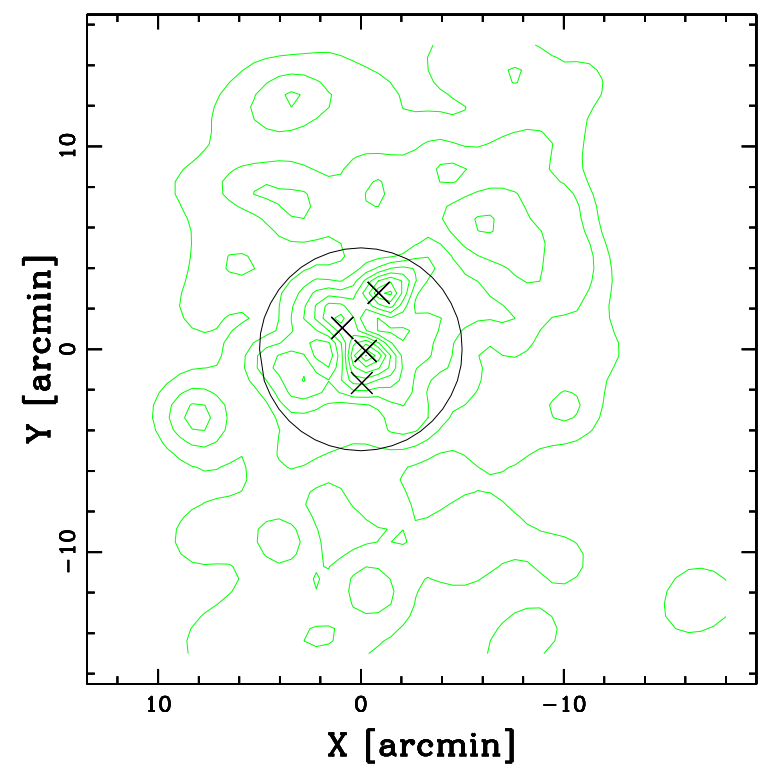

Fig. 8. Spatial distribution on the sky and relative isodensity contour map of likely cluster members with $r^{\prime} \leq 20$, obtained with the 2D DEDICA method. The large faint circle indicate the region of 5'-radius which is sampled by the spectroscopic data. The crosses indicate the position of the peaks in the distribution of the spectroscopic members.

Table 2. Substructure from the photometric sample with $r^{\prime} \leq 20$ found with DEDICA.

\begin{tabular}{lccccc}
\hline \hline Subclump & $N_{\mathrm{S}}$ & $\begin{array}{c}\alpha(\mathrm{J} 2000), \delta(\mathrm{J} 2000) \\
\mathrm{h}: \mathrm{m}: \mathrm{s},{ }^{\circ}:^{\prime}:{ }^{\prime \prime}\end{array}$ & $\rho_{\mathrm{S}}$ & $\chi_{\mathrm{S}}^{2}$ & $\begin{array}{c}\operatorname{dist}(\mathrm{BCG}) \\
h_{70}^{-1} \mathrm{Mpc}\end{array}$ \\
\hline 2D-C & 73 & $053224.6,-113252$ & 1.00 & 31 & $0.07(\mathrm{CBCG})$ \\
2D-NNW & 58 & $053220.4,-112949$ & 0.85 & 25 & $0.08(\mathrm{BCG})$ \\
2D-NE & 36 & $053229.8,-113107$ & 0.66 & 20 & $0.05(\mathrm{BCG})$ \\
\hline
\end{tabular}

Figure 8 shows the contour map for the 902 galaxies selected on the base of the cluster red sequence having $r^{\prime} \leq 20$. The most significant peaks coincide with the peak in the cluster core, the NNW peak, and the NE peak detected in the 2D distribution of spectroscopic members. Similar results are found for the 1304 objects with $r^{\prime} \leq 21$.

As for the results with $r^{\prime} \leq 20$, Table 2 lists the number of assigned members, $N_{\mathrm{S}}$ (Col. 2); the peak position (Col. 3); the density (relative to the densest peak), $\rho_{\mathrm{S}}$ (Col. 4); the value of $\chi^{2}$ for each peak, $\chi_{\mathrm{S}}^{2}$ (Col. 5); the distance of the closest BCG from the peak position with the name of the respective BCG (Col. 6). Ramella et al. (2007) tested the 2D DEDICA procedure on Monte-Carlo simulations reproducing galaxy clusters. They show that the physical significance, i.e. the significance which takes into account the noise fluctuations, associated to the subclusters depends on the statistical significance of the subcluster (recovered from the $\chi_{\mathrm{S}}^{2}$ value) and can be computed using simulations. Comparing the values for the three peaks in A545 with Fig. 2 by Ramella et al. (2007, obtained from simulations of 900 data points) we conclude that these substructures are less than $7 \%$ of probability to be statistical artifacts. In our analysis other peaks have $\chi_{\mathrm{S}}^{2}<17$ and thus a probability larger than $10 \%$ to be artifacts.

To further probe the robustness of these detections, we also apply the Voronoi Tessellation and Percolation (VTP) technique (e.g. Ramella et al. 2001; Barrena et al. 2005). This technique is non-parametric and does not smooth the data. As a consequence, it identifies galaxy structures irrespective of their shapes. The

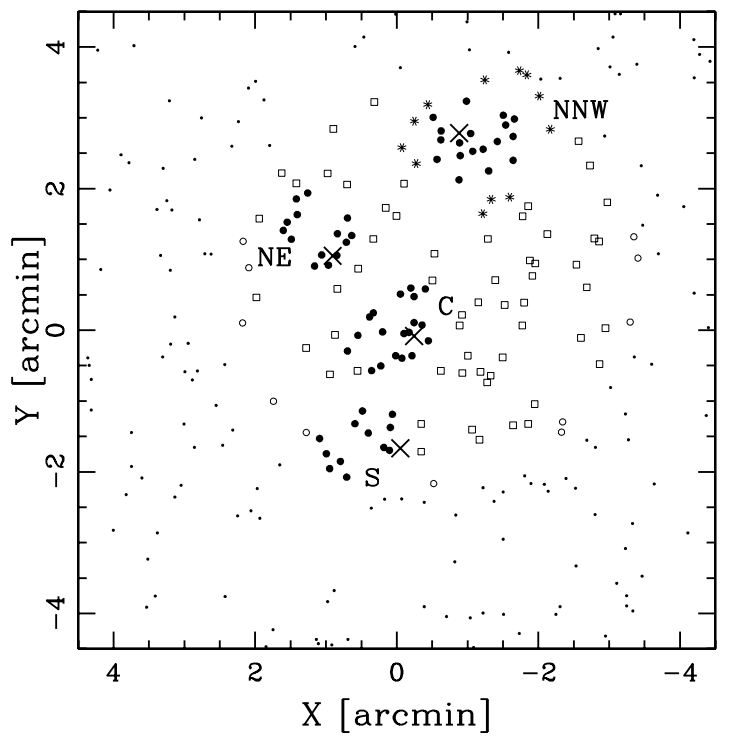

Fig. 9. Galaxies belonging to structures as detected by the Voronoi Tessellation and Percolation technique. The algorithm is run on the sample of likely members with $r^{\prime} \leq 21$ (see text). Open circles, open squares, asterisks and solid circles indicate galaxies in structures at the $90 \%, 95 \%, 98 \%$ and $99 \%$ c.ls., respectively. The C, NE, NNW and S subclumps are indicated. As a comparison, big crosses indicate the positions of the peaks in the distribution of the spectroscopic members.

result of the application of VTP on the sample of 1304 likely members with $r^{\prime} \leq 21$ is shown in Fig. 9. VTP is run four times adopting four detection thresholds: galaxies identified as belonging to structures at $90 \%, 95 \%, 98 \%$ and $99 \%$ c.ls. are shown as open circles, open squares, asterisks and solid circles respectively. VTP confirms the central peak and the NE and NNW ones found by DEDICA in the photometric sample. However, in this case VTP also finds a significant peak located 2' South of the cluster center, roughly coincident with the peak detected by DEDICA in the distribution of spectroscopic members. As for the detection of the southern peak, the difference between DEDICA and VTP applied to the same sample of photometric members can be explained by the fact that DEDICA, like any kernel-based technique in general, is more sensitive to symmetric structures than asymmetric ones. The result is that the southern overdensity, which is very close and less dense than the central one, causes only a southern elongation of the iso-density contours in the DEDICA central peak (see Fig. 8, also more evident in the corresponding figure for $r^{\prime} \leq 21$ ). On the contrary, the non-parametric nature of VTP favors an easier separation of the central and southern peaks (cf. Fig. 9).

\subsection{D-analysis}

Here we use different approaches to analyze the structure of A545 combining position and velocity information.

To search for a possible physical meaning of the three subclusters determined by the two weighted gaps, we compare two by two the spatial galaxy distributions of GV1, GV3, and GV4; GV2 is not considered since is only formed by two galaxies. We find no difference according to the the 2D Kolmogorov-Smirnov test (Fasano \& Franceschini 1987).

We analyze the presence of a velocity gradient performing a multiple linear regression fit to the observed velocities with respect to the galaxy positions in the plane of the sky (e.g., 
den Hartog \& Katgert 1996; Girardi et al. 1996; Boschin et al. 2004). To assess the significance of this velocity gradient we perform 1000 Monte Carlo simulations by randomly shuffling the galaxy velocities and for each simulation we determine the coefficient of multiple determination $\left(R C^{2}\right.$, see e.g., NAG Fortran Workstation Handbook 1986). We define the significance of the velocity gradient as the fraction of times in which the $R C^{2}$ of the simulated data is smaller than the observed $R C^{2}$. We find that the velocity gradient is not significant.

In order to check for the presence of substructure, we combine velocity and position information by computing the $\Delta$-statistics devised by Dressler \& Schectman (1988, hereafter DS-test), which is recommended by Pinkney et al. (1996) as the most sensitive 3D test. For each galaxy, the deviation $\delta$ is defined as $\delta^{2}=\left[\left(N_{\mathrm{nn}}+1\right) / \sigma_{V}^{2}\right]\left[\left(\bar{V}_{1}-\bar{V}\right)^{2}+\left(\sigma_{V, 1}-\sigma_{V}\right)^{2}\right]$, where the subscript "l" denotes the local quantities computed over the $N_{\mathrm{nn}}=10$ neighbors of the galaxy. $\Delta$ is the sum of the $\delta$ of the individual $N$ galaxies and gives the cumulative deviation of the local kinematical parameters (mean velocity and velocity dispersion) from the global cluster parameters. We find $\Delta=108$. The significance of substructure is again checked by running 1000 Monte Carlo simulations, randomly shuffling the galaxy velocities. We find that 336 simulated clusters show a value of the $\Delta$ parameter larger than that of the real cluster leading to non significant presence of substructure.

Following Pinkney et al. (1996; see also Ferrari et al. 2003), we apply other two classical 3D tests: the $\epsilon$-test (Bird 1993) based on the projected mass estimator and the centroid shift or $\alpha$-test (West \& Bothun 1990). The details of these tests can be found in the above papers: we only point out that we consider ten as the number of the nearest neighbors for each galaxy and we use the above Monte Carlo simulations to compute the substructure significance. In both cases we do not find evidence for significant substructure.

We also consider two kinematical estimators alternative to the $\delta$ parameter of the DS-test, i.e. we consider separately the contributes of the local mean $\left.\delta_{V}^{2}=\left[\left(N_{\mathrm{nn}}+1\right) / \sigma_{V}^{2}\right]\left(\bar{V}_{1}-\bar{V}\right)^{2}\right]$, and dispersion $\left.\delta_{\mathrm{s}}^{2}=\left[\left(N_{\mathrm{nn}}+1\right) / \sigma_{V}^{2}\right]\left(\sigma_{V, 1}-\sigma_{V}\right)^{2}\right]$ (see, e.g. Girardi et al. 1997, Ferrari et al. 2003). For both of the above estimators, we do not find evidence for significant substructure.

\section{X-ray morphological and spectral analysis}

We retrieved from the XMM-Newton archive the available observation of A545 (Obs. ID. 0304750101), which has a nominal exposure time of $36.4 \mathrm{ks}$. We reprocessed the Observation Data Files (ODF) using the Science Analysis System (SAS) version 9.0. After the production of the calibrated event lists for the EPIC MOS1, MOS2 and pn observations with the emchain and epchain tasks, we tried to perform a soft proton cleaning. Unfortunately, by screening the light curves, produced in $100 \mathrm{~s}$ bins in the 10-12 keV band, we discovered that this observation is highly affected by soft protons flares. The contamination is so severe that we could not apply the standard count rate thresholds for the MOS and $p n$ (i.e., $0.15 \mathrm{cts} / \mathrm{s}$ and $0.35 \mathrm{cts} / \mathrm{s}$, respectively). We decided to apply higher thresholds, namely $1.6 \mathrm{cts} \mathrm{s}^{-1}$ for MOS1 and MOS2 and $4.5 \mathrm{cts} \mathrm{s}^{-1}$ for $p n$, and then use these least contaminated data to extract global X-ray properties in the highest surface brightness cluster regions only, where the ICM photons clearly dominate over the soft proton background. We also filtered event files according to FLAG $($ FLAG $=0)$ and PATTERN $($ PATTERN $\leq 12$ for MOS and PATTERN $=0$ for $p n$ ) criteria. The resulting effective exposure time of the observation is $16 \mathrm{ks}$ for the MOS and $9 \mathrm{ks}$ for the $p n$.

\subsection{Surface brightness analysis}

The EPIC (i.e. with all the three detectors summed together) surface brightness contours of A545 in the 0.4-2 keV band, overplotted to the INT $r^{\prime}$-band optical image, are shown in Fig. 1. We produce the X-ray image following the procedure described in Rossetti et al. (2007, see their Fig. 6).

The analysis of the surface brightness map reveals that the cluster is strongly elongated NNW-SSE. Following the procedure of Hashimoto et al. (2007), we compute the ellipticity of the isophotes. We find that the cluster is indeed strongly elongated, with an ellipticity $\epsilon=1-b / a=0.37$, where $a$ and $b$ are the major and minor axes of the isophotes, respectively.

To produce the surface-brightness profile (see Eckert et al. 2011), we extract MOS1 and MOS2 count images in a broad band (0.4-8 keV), to include as many photons as possible, and produce the corresponding exposure maps using the SAS tool eexpmap to correct for vignetting effects. Point sources are excised from the image using a local background method. Namely, we excise regions of the image where the observed count rate is at least 5 sigma above the local background. We then extract the surface-brightness profile of the cluster centered on the emission centroid in elliptical annuli, with bins of width 7". More specifically, we select regions with equal elliptical distance to the center, where the distance is given by $R=\sqrt{\frac{a^{2}}{b^{2}} x_{1}^{2}+x_{2}^{2}}, x_{1}$ and $x_{2}$ being the distances along the major and minor axes. The profile is then fitted by a standard $\beta$ model (Cavaliere \& Fusco-Femiano 1976),

$S B(R)=S_{0}\left[1+\left(R / R_{\mathrm{c}}\right)^{2}\right]^{-3 \beta+1 / 2}+B$,

where $R_{\mathrm{c}}$ is the core radius along the major axis, $\beta$ gives the slope of the outer density profile, and $B$ is a constant to determine the background level. The surface brightness profile extracted in elliptical annuli is rather well represented $\left(\chi_{\text {red }}^{2}=112.5 / 73\right)$ by a beta profile with $\beta=0.87_{-0.02}^{+0.03}$ and a core radius $R_{\mathrm{c}}=2.03 \pm$ $0.06^{\prime}\left(333 \pm 10 h_{70}^{-1} \mathrm{kpc}\right)$ along the major axis, which corresponds to a core radius of $1.28 \pm 0.04^{\prime}\left(210 \pm 7 h_{70}^{-1} \mathrm{kpc}\right)$ along the minor axis (this profile is plotted as a blue line in Fig. 11).

We look for substructures in A545 by removing the bestfit elliptical beta model from the surface brightness image. The residuals, in units of standard deviation per pixel, are shown in Fig. 10, with radio contours overlaid. At $\sim 2.7^{\prime}\left(\sim 440 h_{70}^{-1} \mathrm{kpc}\right)$ North of the X-ray centroid, we note a significant depletion (or "jump") with respect to the average profile, that could be a signature of a cold front or of a shock (see also Fig. 10). To investigate this possibility, we extract the surface brightness profile in a sector with position angles 70-120 deg (with respect to the RA axis; the sector is shown in Fig. 10). In Fig. 11 we plot the surface brightness profile in this particular sector, compared to the average profile of the whole cluster, described above. To characterize more quantitatively the jump we compare the slope of the mean profile with the slope found in profile of the 70-120 deg sector by modeling both profiles in the radial range $100^{\prime \prime}-200^{\prime \prime}$ with a simple power law model. We find that the difference of the slope estimated in the 70-120 deg sector with respect to the slope from the mean profile is $0.77 \pm 0.11$.

Other interesting features noticeable from the residuals shown in Fig. 10 are the asymmetry along the E-W axis, the $\mathrm{W}$ side showing an excess of emission compared to the $\mathrm{E}$, and a 


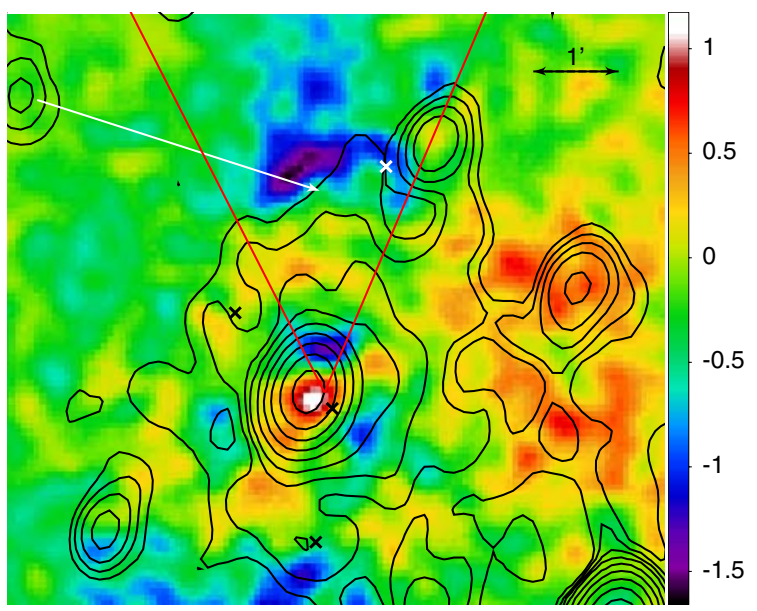

Fig. 10. Residuals of the X-ray surface brightness map after subtraction of the best fitting elliptical beta model (the colors correspond to the deviations in sigma to the surface brightness profile for each pixel). The black contours show the extended radio emission (Bacchi et al. 2003). The arrow indicates the position of the North surface brightness jump. The two red lines show the $70-120$ deg sector used to produce the profile plotted in Fig. 11. Crosses show the positions of the peaks in the distribution of the optical spectroscopic members as in Figs. 8, and 9 .

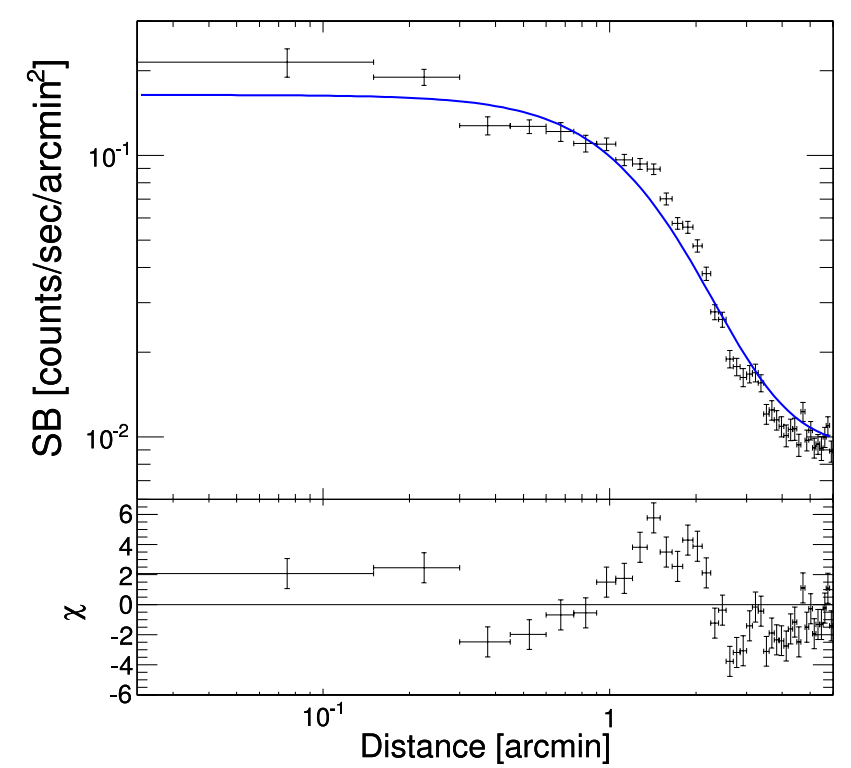

Fig. 11. XMM-Newton surface brightness profile in direction of the North feature (from a sector with position angles 70-120 deg). The blue line is the best-fitting single beta model for the total profile. At $\sim 2.7^{\prime}$ from the center there is a clear surface brightness jump.

surface brightness decrement $\sim 30^{\prime \prime}$ North of the emission peak. While the E-W asymmetry might be worthwhile to be investigated with new XMM-Newton data of better quality, the decrement right $\mathrm{N}$ of the $\mathrm{X}$-ray emission peak could be due to an imperfect subtraction of a point-like source (at RA $=05^{\mathrm{h}} 32^{\mathrm{m}} 24^{\mathrm{s}} .91$ and Dec $\left.=-11^{\circ} 31^{\prime} 399^{\prime \prime} 97\right)$ and it would need the higher resolution of a Chandra observation to be investigated in details.

\subsection{Spectral analysis}

We apply the background subtraction using blank-sky fields for EPIC MOS and $p n$ that were produced by Leccardi et al. (2008, see Appendix B in their paper) by analyzing a large number of observations for a total exposure time of $\sim 700 \mathrm{ks}$ for MOS and $\sim 500 \mathrm{ks}$ for $p n$. To take into account temporal variations of the background, more than possible in this highly flared observation, we also perform a background rescaling by estimating the background intensity in this observation from a spectrum extracted in an external annulus between $10^{\prime}$ and $12^{\prime}$ centered on the emission peak in the 10-12 keV band (to avoid possible extended cluster emission residuals in this region). We then rescale the blank-sky fields background to the local value.

Using the XSPEC package (version 11.3.2, Arnaud et al. 1996), we analyze the EPIC spectrum of a circular region of $310 \mathrm{kpc}$ radius centered on the X-ray peak (we exclude the pointlike source North of the center). We model the spectrum with a one temperature thermal model with the plasma in collisional ionization equilibrium (mekal model in the XSPEC nomenclature), multiplied by the Galactic hydrogen column density, $N_{\mathrm{H}}=1.02 \mathrm{E}+21 \mathrm{~cm}^{-2}$, determined by HI surveys (Kalberla et al. 2005) through the wabs absorption model. The redshift is fixed to $z=0.158$. The $\mathrm{Fe}$ abundance is measured relative to the solar photospheric values of Anders \& Grevesse (1989), where $\mathrm{Fe}=4.68 \times 10^{-5}$. We find that the ICM has a global temperature of $8.08 \pm 0.32 \mathrm{keV}$ and $\mathrm{Fe}$ abundance of $0.30 \pm 0.05$ (solar units).

We apply the same analysis to the spectrum extracted from a circular region of radius $0.05 r_{180}=0.6^{\prime} \quad(105 \mathrm{kpc})$ centered on the X-ray emission peak, which is the dimension of a typical cool core region (e.g., Leccardi et al. 2010). The resulting temperature does not show any significant decrement within the errors $(8.42 \pm 0.48 \mathrm{keV})$, as expected for a non cool core cluster.

We also tried to provide a more detailed description of the temperature and abundance structure of A545. For example, investigating differences of surface brightness, temperature and abundance in regions coincident with the optical subclumps, or producing thermodynamical maps (e.g. temperature, pressure, entropy) using the procedure described in Rossetti et al. (2007). However, the significant residual soft protons contamination the background of the "cleaned" dataset is a factor of 4 larger than that of a normal observation - prevents us from providing this more detailed description of A545.

\section{Discussion and conclusions}

We have analyzed for the fist time the internal dynamics of A545. We find $\langle z\rangle=0.1580 \pm 0.0004$, high values of the velocity dispersion $\sigma_{V}=1220_{-68}^{+82} \mathrm{~km} \mathrm{~s}^{-1}$ and X-ray temperature $k T_{\mathrm{X}}=8.08 \pm 0.32 \mathrm{keV}$. These results are comparable to the highest values found in typical clusters (see Mushotzky \& Scharf 1997; Girardi \& Mezzetti 2001). Our estimates of $\sigma_{V}$ and $T_{\mathrm{X}}$ are fully consistent when assuming the equipartition of energy density between ICM and galaxies. We obtain $\beta_{\mathrm{spec}}=1.12_{-0.12}^{+0.15}$ to be compared with $\beta_{\text {spec }}=1$, where $\beta_{\text {spec }}=\sigma_{V}^{2} /\left(k T_{\mathrm{X}} / \mu m_{\mathrm{p}}\right)$ with $\mu=0.58$ the mean molecular weight and $m_{\mathrm{p}}$ the proton mass (see also Fig. 5).

In the framework of usual assumptions (cluster sphericity, dynamical equilibrium, coincidence of the galaxy and mass distributions), one can compute virial global quantities. Following the prescriptions of Girardi \& Mezzetti (2001, Eq. (1) with the scaling of $H(z)$ ), we assume for the radius of the quasi-virialized region is $R_{\text {vir }}=0.17 \times \sigma_{V} / H(z)=2.74 h_{70}^{-1} \mathrm{Mpc}$, see also Eq. (8) of Carlberg et al. (1997) for $R_{200}$. We compute the virial mass (Limber \& Mathews 1960; see also, e.g., Girardi et al. 1998):

$M=3 \pi / 2 \cdot \sigma_{V}^{2} R_{\mathrm{PV}} / G-\mathrm{SPT}$, 
where SPT is the surface pressure term correction (The \& White 1986 ) and $R_{\mathrm{PV}}$ is a projected radius (equal to two times the projected harmonic radius).

The estimate of $\sigma_{V}$ is robust when computed within a large cluster region (see Fig. 5). The value of $R_{\mathrm{PV}}$ depends on the size of the sampled region and possibly on the quality of the spatial sampling (e.g., whether the cluster is uniformly sampled or not). Since in A545 we sample only a fraction of $R_{\text {vir }}$, we have to use an alternative estimate of $R_{\mathrm{PV}}$ on the basis of the knowledge of the galaxy distribution. Following Girardi et al. (1998; see also Girardi \& Mezzetti 2001), we assume a King-like distribution with parameters typical of nearby/medium-redshift clusters: a core radius $R_{\mathrm{c}}=1 / 20 \times R_{\mathrm{vir}}$ and a slope-parameter $\beta_{\text {fit,gal }}=0.8$, i.e. the volume galaxy density at large radii goes as $r^{-3 \beta_{\text {fitgal }}}=r^{-2.4}$. We obtain $R_{\mathrm{PV}}\left(<R_{\mathrm{vir}}\right)=2.04 h_{70}^{-1} \mathrm{Mpc}$, where a $25 \%$ error is expected (Girardi et al. 1998, see also the approximation given by their Eq. (13) when $A=R_{\text {vir }}$ ). The value of SPT depends strongly on the radial component of the velocity dispersion at the radius of the sampled region and could be obtained by analyzing the (differential) velocity dispersion profile, although this procedure would require several hundreds of galaxies. We decide to assume a $20 \%$ SPT correction as obtained in the literature by combining data on many clusters sampled out to about $R_{\text {vir }}$ (Carlberg et al. 1997; Girardi et al. 1998). We compute $M_{\text {one-cluster }}\left(<R_{\text {vir }}=2.74 h_{70}^{-1} \mathrm{Mpc}\right)=2.7_{-0.7}^{+0.8} \times 10^{15} h_{70}^{-1} M_{\odot}$.

\subsection{Internal structure}

The appearance of this cluster is well far from being that of a relaxed cluster. Evidence of this comes from both optical and $\mathrm{X}$-ray analyses.

The position of the highest peak of the 2D galaxy distribution coincides with the position of the peak in the X-ray distribution, but other two, maybe three, galaxy clumps are detected. One is located $\sim 3^{\prime}$ at North, slightly NNW (close to the BCG3), and seems a well separated unit from the cluster core. The other is located $\sim 2^{\prime}$ at NE, with isodensity contours elongated toward to the BCG4. The possible, fourth peak (detected by the VTP analysis) lies at South. Very interestingly, the southern clump, if real, well delineates with the NNW clump a possible direction for the cluster accretion (see Figs. 6 and 9). Another possible direction for the cluster accretion is the NE-SW direction as also suggested by the apparent SW extension of the radio halo at large scale (see Fig. 2 of Bacchi et al. 2003) and the small scale features concerning the star pile (see Fig. 1 of S07, see also the insect figure in Fig. 2 and Sect. 5.3).

The integral velocity dispersion profile increases from the central cluster regions out to $0.1 h_{70}^{-1} \mathrm{Mpc}$ (see Fig. 5, bottom panel). Although an increase in the velocity-dispersion profile in cluster central regions might be due to dynamical friction and galaxy merging (e.g., Menci \& Fusco-Femiano 1996; Girardi et al. 1998; Biviano \& Katgert 2004), in the case of the substructured cluster A545 it is likely induced by the contamination of the galaxies of a secondary clump, i.e. to the mix of galaxies of two systems having different mean velocities (e.g., Girardi et al. 1996; Barrena al. 2007b). The increase of $\sigma_{V}$ due to the contamination starts well before the inclusion of (the center of) the companion system, e.g. leading to "uncontaminated" regions of $0.3-0.4 h_{100}^{-1} \mathrm{Mpc}$ for rich clusters having a companion at $1 h_{100}^{-1}$ Mpc (see A3391 and A3395 in Fig. 1 of Girardi et al. 1996). Assuming a similar scenario for A545, the increase of the $\sigma_{V}$ profile in the central region shown by Fig. 5 is due to the via via increasing contamination of the close subclumps (note that the NE clump is only $\sim 0.3 h_{70}^{-1} \mathrm{Mpc}$ far from the C clump). We take the value of the velocity dispersion $\sigma_{V} \sim 700 \mathrm{~km} \mathrm{~s}^{-1}$, based on the five most central galaxies within $0.05 h_{70}^{-1} \mathrm{Mpc}$ (i.e. the first left point plotted in Fig. 5, bottom panel), as the most representative value of the velocity dispersion of the "uncontaminated" main C subcluster. Following the above virial mass estimation procedure or, simpler, applying the usual scaling-laws where $R_{\text {vir }} \propto \sigma_{V}$ and $M\left(<R_{\text {vir }}\right) \propto \sigma_{V}^{3}$, we use the value of $\sigma_{V} \sim 700 \mathrm{~km} \mathrm{~s}^{-1}$ to estimate the virial mass of $\mathrm{C}$ subcluster as $M_{\mathrm{C}}\left(<R_{\mathrm{vir}} \sim 1.6 h_{70}^{-1} \mathrm{Mpc}\right) \sim 0.5 \times 10^{15} h_{70}^{-1} M_{\odot}$.

We also analyze the $\sigma_{V}$-profiles corresponding to the NNW and NE galaxy clumps, but there is no trace of a increase of the $\sigma_{V}$-profiles, rather the central values of $\sigma_{V}$ are very high $\left(>1500 \mathrm{~km} \mathrm{~s}^{-1}\right.$ ) suggesting that these groups are already contaminated in their central regions and cannot be detected as separate units. This result prevents us to attempt an alternative estimate of the whole A545 complex as the combination of the three subclusters directly from the kinematics of galaxies.

We can attempt an alternative procedure using galaxy number as a proxy for the mass. According to our analysis of the 2D substructure (Table 2), we can compute the mass of the cluster from the three main clumps as $M_{\text {sum-subclusters }}(R<$ $\left.1.6 h_{70}^{-1} \mathrm{Mpc}\right)=M_{\mathrm{C}} \cdot N_{\mathrm{C}+\mathrm{NNW}+\mathrm{NE}} / N_{\mathrm{C}}=0.5 \cdot 167 / 73 \sim$ $1.1 \times 10^{15} h_{70}^{-1} M_{\odot}$. Since we do not consider the likely southern subcluster, this value can be taken as a lower limit for the mass estimate. To rescale the value of $M_{\text {one-clusterwe assume }}$ that the system is described by a King-like mass distribution (see above) or, alternatively, a NFW profile where the massdependent concentration parameter $c$ is taken from Navarro et al. (1997) and rescaled by the factor $1+z$ (Bullock et al. 2001; Dolag et al. 2004). We obtain $M_{\text {one-cluster }}\left(R<1.6 h_{70}^{-1} \mathrm{Mpc}\right) \sim$ (1.7-1.8) $\times 10^{15} \quad h_{70}^{-1} M_{\odot}$. Therefore, we estimate the range for the mass of A545 as $M\left(R<1.6 h_{70}^{-1} \mathrm{Mpc}\right)=(1.1-$ 1.8) $\times 10^{15} h_{70}^{-1} M_{\odot}$. Note that, assuming an isothermal $\beta$ model for the ICM (e.g., Eq. (6) of Henry et al. 1993) and using the global ICM temperature and the fitted parameters of the $\beta$ model of Sect. 4, we obtain from X-ray data a mass $M_{\mathrm{X}}(R<$ $\left.1.6 h_{70}^{-1} \mathrm{Mpc}\right) \sim 1.3 \times 10^{15} h_{70}^{-1} M_{\odot}$, falling in the estimated mass range. According to the usual scalings, the above mass range leads to these global virial quantities for A545: $\sigma_{V} \sim(900$ 1200) $\mathrm{km} \mathrm{s}^{-1}$ and $M_{\mathrm{vir}}\left(<R_{\mathrm{vir}}=2-2.7 h_{70}^{-1} \mathrm{Mpc}\right)=(1.5-$ 2.7) $\times 10^{15} h_{70}^{-1} M_{\odot}$.

As for the view angle of the merger axis, we have three indications that the merger is mostly occurring in the plane of the sky: i) the successful detection of substructure mostly through the $2 \mathrm{D}$ analysis suggests that the intervening subclusters are very close in the velocity space (see Pinkney et al. 1996); ii) the mean velocity profile of the $\mathrm{C}$ galaxy clump shows an increase in the central region, likely due to the contamination of close clumps, but quite modest $\sim 500 \mathrm{~km} \mathrm{~s}^{-1}$ (Fig. 5, central panel); iii) the likely presence of a shock in the ICM (see below) since a shock is more easily discernible in an X-ray image when the cluster merger occurs in the plane of the sky (e.g., Markevitch 2010).

Even in the case of very unrelaxed clusters, the brightest galaxies seem to trace the galaxy or dark matter subclumps both in two dimensions (Beers \& Geller 1983) and in 3D space (e.g. Abell 520, Girardi et al. 2008). In A545 the brightest galaxies BCG1, BCG2, BCG3, and BCG4 avoid the cluster core and are far from the mean cluster velocity, but the BCG3 and BCG4 seem to be related with two peaks in the galaxy distribution (NNW and NE clumps). Instead, the brightest galaxy in the cluster core $(\mathrm{CBCG})$, although is more than 1 mag fainter then the 
BCG1, is related to the main density peak and is at rest with respect to the whole cluster rest frame.

The indications that we derive from the analysis of the $\mathrm{X}$-ray surface brightness distribution (namely: the deviations from spherical symmetry, the northern discontinuity, the western excess) clearly provide evidence of a disturbed dynamical phase, too. A545 is indeed strongly elongated, with an ellipticity $\epsilon=0.37$. From the sample of Hashimoto et al. (2007), we see that such a value would classify A545 as one of the most elliptical objects. Since the authors note a correlation between the ellipticity and the dynamical state, this supports a scenario where the cluster is dynamically active. Moreover, the elongation is mostly in the NNW-SSE direction indicating the same direction of the NNW galaxy clump with respect to cluster center. Another hint on the unrelaxed state of A545 comes also from the clear twisting of the isophotes observable from the center towards the more external regions (see Fig. 1). As for the western $\mathrm{X}$-ray excess, very noticeably the $2 \mathrm{D}$ galaxy distribution reveals an excess in the western regions, too. In fact, looking at Fig. 9, we note that the distribution of galaxies assigned to the cluster with a probability of $\gtrsim 95 \%$ ( $\gtrsim 90 \%)$ do not follow that of galaxies with highest probabilities, neither is symmetric around the cluster center. The result is that, out of galaxies with a probability of $\gtrsim 95 \%$ ( $\gtrsim 90 \%$ ), we find 13 (18) galaxies $2^{\prime}$ west from the cluster center - where we start to detect the X-ray excess - and no (3) galaxies $2^{\prime}$ east from the cluster center.

The most interesting feature in the X-ray surface brightness is the northern sharp discontinuity $\left(\sim 2.7^{\prime}\right.$ with respect to the X-ray centroid), which is a signature of a shock or a cold front (e.g., Markevitch 2010, and refs. therein). Interestingly, the shock possibility is supported by the fact that at the location of the surface brightness jump the giant radio halo also shows an edge (see Fig. 10), as it has been recently found in other merging clusters (Markevitch 2010). However, only an ICM temperature analysis across the surface brightness discontinuity could allow us to discriminate between the two possibilities, and with the present dataset this analysis is not feasible because of the high soft protons background.

A shock front generated during a cluster merger is expected to lie perpendicular to the merging axis: see, e.g., the first known case of 1E 0657-56 (Markevitch et al. 2002) and numerical simulations (e.g. by Springel \& Farrar 2007). As for the clusters we have already analyzed in the context of the DARC project, the shock front in Abell 520 (Markevitch et al. 2005) is perpendicular to the NE-SW direction of the main merger (Markevitch et al. 2005; Girardi et al. 2008); the likely shock front detected in Abell 2219 (Million \& Allen 2009) is perpendicular to the SE-NW direction of the merger (Boschin et al. 2004); the shock front in Abell 2744 is perpendicular to the SSE-NNW direction of the merger (Owers et al. 2011), somewhat a more southeasterly direction than that suggested by Boschin et al. (2006). As for A545, we note that the discontinuity is roughly perpendicular to the bisecting line of the two likely merging directions (i.e. towards NNW and towards NE, see Fig. 6). This finding reminds the case of Abell 2345 - hereafter A2345 - where the western relic, likely generated by a shock, is found to be perpendicular to the bisecting of the two merging directions (Boschin et al. 2010). Note that, to date, no shock is detected in A2345 and we must rely on the position of the relic itself. Indeed, most relics are located far from the X-ray bright cluster regions, so it is rarely possible to check for the presence of a shock front in a X-ray image, and even more difficult to get a confirming temperature measurement, a likely exception being Abell 3667 by Finoguenov et al. (2010). Although A2345 and A545 have both a complex merging structure which can help us to understand the position and geometry of the respective likely-shock/relic, the structure of these two clusters differ in two maior points. The subclusters are much closer in A545 than in A2345 (by roughly a factor two, cf. Fig. 6 with Fig. 7 of Boschin et al. 2010) suggesting a different merging age and, indeed, we below give a lower estimate for the time elapsed since the collision with respect to A2345. Second, the main subcluster is richer - with respect to other subclusters - in A545 than in A2345 (cf. the number of assigned galaxies by 2D DEDICA, $N_{\mathrm{S}}$ in Table 2 and Table 2 of Boschin et al. 2010).

Summarizing, we find that A545 is formed by three, likely four, subclusters and infer two directions for the accretion path (NNW-SSE and NW-SE directions). We determine the (projected) position of the subclusters and give some indications about their relative importance and the angle of view of the merger. These results overcome the gross-scale result of an elongation in the ICM (Buote \& Tsai 1996). Moreover, Buote \& Tsai gave no information about the merge age and, indeed, also assuming a post-collision phase, the elongation of the X-ray emission is a long-lived phenomenon (up to $5 \mathrm{Gyr}$ after the core passage; see Roettiger et al. 1996). More precise information can be derived from the northern discontinuity we observe in the X-ray surface brightness, if confirmed to be due to a shock. In fact, we can estimate the velocity of the shock front $v_{\mathrm{s}}$ from $\mathcal{M}=v_{\mathrm{s}} / c_{\mathrm{s}}$ (e.g., Sarazin 2002), where $\mathcal{M}$ is the Mach number of the detected shock - typically $\mathcal{M} \sim 2$ in clusters with radio halos (Markevitch et al. 2010) - and $c_{\mathrm{S}}$ is the sound speed in the preshock gas $-c_{\mathrm{s}} \sim 1150 \mathrm{~km} \mathrm{~s}^{-1}$ from the thermal velocity (i.e. from the observed $k T_{\mathrm{X}}$ in the $\beta_{\text {spec }}=1$ assumption). We obtain $v_{\mathrm{s}} \sim 2300 \mathrm{~km} \mathrm{~s}^{-1}$. When using the distance of the $\mathrm{X}$-ray surface brightness discontinuity from the cluster center, $r_{\text {shock }} \sim 0.45 h_{70}^{-1} \mathrm{Mpc}$, we estimate that $t=r_{\text {shock }} / v_{\mathrm{s}} \sim 0.2 \mathrm{Gyr}$ is the time elapsed since the collision.

\subsection{A545 and predictions of halo/relic models}

As in the case of other clusters of DARC study, A545 supports the scenario that extended, diffuse radio emissions are associated to dynamically disturbed clusters, where major merging phenomena are occurring.

More quantitatively, we can use our results to re-examine A545 with respect to the observed scaling relations among $P_{1.4 \mathrm{GHz}}$, the halo radio power, and $M_{\mathrm{H}}$, the total cluster mass contained within the size of the radio halo, $R_{\mathrm{H}}$, and the virial radius (Cassano et al. 2007). Rescaling the A545 mass to $R_{\mathrm{H}}=$ $380 h_{70}^{-1} \mathrm{kpc}$ (Cassano et al. 2007), we obtain $M_{\mathrm{H}}\left(<R_{\mathrm{H}}=\right.$ $\left.0.38 h_{70}^{-1} \mathrm{Mpc}\right) \sim(0.2-0.4) \times 10^{15} h_{70}^{-1} M_{\odot}$. This is larger than the value of $0.12 \times 10^{15} h_{70}^{-1} M_{\odot}$ reported by Cassano et al., large part of the difference being due to the small value assumed for the X-ray temperature $\left(k T_{\mathrm{X}}=5.5 \mathrm{keV}\right.$ by David et al. 1993; Cassano et al. 2006). The result is that the position of A545 moves from under to above the $M_{\mathrm{H}}-R_{\mathrm{H}}$ relation and from the left to the right of the $P_{1.4 \mathrm{GHz}}-M_{\mathrm{H}}$ relation (Figs. 8 and 9 of Cassano et al. 2007). This example points out the importance of re-examining global cluster dynamical quantities to fix observational scaling relations, in particular since these relations are claimed to be predicted on the basis of the re-acceleration model (Cassano et al. 2007). When looking at the $R_{\mathrm{H}}-R_{\text {vir }}$ relation obtained through numerical simulations (Donnert et al. 2010), A545 can be used, as other clusters listed by Cassano et al. (2007), to reject the proposed hadronic model where the energy density of the cosmic ray protons is taken as a constant 
fraction of the thermal energy density population, $X_{\mathrm{CR}}=$ const., but not to check other two variations of the basic model, i.e. using a radius dependent $X_{\mathrm{CR}}$ and flattening the simulated magnetic field to be $B \propto \sqrt{\rho}$ with $\rho$ the mass density, where more massive clusters would be useful (see Fig. 10 of Donnert et al. 2010).

We can also consider A545 with respect to the relation between $\alpha_{\text {halo }}$, the spectral index of radio halo, and $r_{\text {relic }}$, the distance of relic (or shock) from the cluster center. This relation is expected in the unified halo-relic hadronic model by Keshet (2010) according to the idea that relic/shock clustercentric distance can be used as a proxy for the time elapsed since the merger. Assuming that the northern discontinuity we observe in the X-ray surface brightness is really due a shock, we obtain $r_{\text {relic }}=r_{\text {shock }} \sim 450 h_{70}^{-1} \mathrm{kpc}$. Considering that $\alpha_{\text {halo }}>1.4$ (Bacchi et al. 2003), A545 well lies in the $r_{\text {relic }}-\alpha_{\text {halo }}$ diagram presented in the literature and only containing, to date, ten clusters (Keshet 2010, Fig. 28).

To better investigate the structure of A545 we asked other time to XMM-Newton (and were approved after the submission of this paper AO-10 - ID.067467 - PI S. De Grandi). We are also planning new spectroscopical observations since for the study of the dynamics of complex merging clusters three times more galaxies need to solve the cluster structure (e.g., in A520 Girardi et al. 2008). Finally, due to our indications for a merger mostly occurring in the plane of sky, we point out to the advantage of having deep photometry and a weak lensing analysis for better determine the mass of the subclusters.

\subsection{Star pile and merging scenario}

The radial velocity of the star pile as measured by S07 is $47100 \pm 60 \mathrm{~km} \mathrm{~s}^{-1}$. We show that the star pile is well at rest within the cluster $\left(\langle v\rangle=47373 \pm 125 \mathrm{~km} \mathrm{~s}^{-1}\right)$ and has the same velocity of the CBCG $\left(47071 \pm 26 \mathrm{~km} \mathrm{~s}^{-1}\right)$. However, the star pile is not symmetric with respect to the CBCG (see our Fig. 2 and Fig. 1 in S07, where the CBCG is indicated by "SGH1"), rather lies at SW with respect to the CBCG. The star pile itself is someway elongated in the NE-SW direction. As for the three faint galaxies embedded in the star pile, the central one (galaxy "g2" in Fig. 2) has a velocity lower by $1300 \mathrm{~km} \mathrm{~s}^{-1}$ than the star pile velocity as found by S07, the western object (the M32like dwarf elliptical cE, galaxy "g1") has a velocity lower by $750 \mathrm{~km} \mathrm{~s}^{-1}$, no redshift is available for the eastern object (galaxy "g3").

The red color of the star pile (S07) and the absence of a clear cool core - the X-ray analysis did not show any evidence of cool core from both the surface brightness profile and the temperature analysis - disfavor the possibility that the star pile is recently created by cooling of the intracluster medium and subsequent star formation. The star pile in A545 might rather be the debris of a tidal stripping or be created through a "dry" merger, i.e., a merger where the progenitor galaxies contain little or no gas such that little star formation is triggered by galaxy merger (e.g., Bell et al. 2006; Liu et al. 2009). The geometry and the velocity of the star pile suggests that it is someway related with the CBCG. Thus a possible source for the star pile is obviously the CBCG itself or rather the compact dwarf $\mathrm{cE}$, likely stripped by interaction with the CBCG. In fact, compact elliptical galaxies are though to form through tidal stripping (Nieto \& Prugniel 1987; Bekki et al. 2001).

In A545 the star pile is likely connected with the formation process of a bright BCG at the cluster center at expenses of other galaxies as suggested by Merritt (1984), and shown in numerical simulations (e.g., Dubinsky 1998; Murante et al. 2007) and in observations (e.g., van Dokkum 2005). In particular, the luminosity of the star pile estimated by S07 corresponds to $L_{r^{\prime}} \sim 1 \times 10^{11} h_{70}^{-2} L_{\odot}$ (using $V-r^{\prime}$ colors for E-type galaxy by Fukugita et al. 1995), i.e. roughly the double of the luminosity of the CBCG. The co-addition of the CBCG, the star pile, and cE will lead to a galaxy brighter than/as bright as the BCG1.

Moreover, in the case of A545, the global environment seems to be critical for the formation of a BCG, too. In fact, the CBCG seems to point towards the NE group with respect to the star pile and the star pile is elongated in the same direction. The relative high velocity of $\mathrm{cE}$ suggests instead that it, if really close to the $\mathrm{CBCG}$, is presently on a radial orbit with respect to the main central group. This agrees with the picture that BCG formation is caused by galaxies falling along radial orbits aligned with the accreting filaments that feed cluster growth (e.g., Dubinski 1998). Thus in A545 both the intracluster light and the following, likely formation of a dominant galaxy seem to be related to an extreme dynamical event such as a cluster merger. This might explain why the intracluster light in A545 is so asymmetric with respect to the CBCG when comparing the star pile with the results of simulations (e.g., Dubinski 1998).

According to theoretical scenarios the hierarchical formation on both galaxy and cluster scales are expected. In particular, BCGs are suggested to grow, at least in large part, via merging with the BCGs of subclusters that have fallen into the cluster, although the rate of grow of BCGs seems slower than that of parent clusters (Lin \& Mohr 2004, and refs. therein). The memory of a connection between BCGs and their parent clusters is likely imprinted on the alignment of BCGs with their hosts (Sastry 1968; Binggeli 1982; Niederste-Ostholt et al. 2010). Recent, detailed studies of clusters hosting BCGs (or central dominant "cD" galaxies) with multiple-nucleus provide important evidence for a scenario where the $\mathrm{BCG} / \mathrm{cD}$ formation is likely due to the cluster merger. In particular, in a few, very interesting cases, the clump of cold, very dense gas centered on the BCG shows a plume aligned with (one of) the merging axis (Abell 3266 by Henriksen \& Tittley 2002; Abell 521 by Ferrari et al. 2006). This gas is explained as due to stripped material related to an early phase of the $\mathrm{BCG} / \mathrm{cD}$ formation, as suggested by the asymmetry in the gas distribution.

A545 is the first case where the connection between the cluster merger and the BCG formation is strongly pointed out by an asymmetric stripped stellar matter aligned with the merging direction. The asymmetry of the star pile indicates that its origin is very recent since $N$-body simulations find that streams of intracluster light found in the core of the clusters, which are characterized by short dynamical times, are quickly destroyed with times $\lesssim 1$ Gyr (Rudick et al. 2009). Present photometric data do not show evidence of a multiple-nucleus in CBGC as, e.g., in Abell 521 (Ferrari et al. 2006) and Abell 3266 (Henriksen \& Tittley 2002) supporting the idea we are looking at a very preliminary phase of the BCG formation. Another case of a very asymmetric intracluster light is that of CL $0958+4702$ at $z=0.39$ but there is no published study about the internal dynamics of this cluster (Rines et al. 2007). In this context, A545 represents a textbook cluster where to study the simultaneous formation of a galaxy system and its brightest galaxy.

After the submission of this paper for publication, Salinas et al. (2011) have presented new deep longslit observations of the star pile using VLT/FORS2 and Gemini/GMOS revealing a very irregular velocity field with parts of the star pile being associated with the g3 galaxy and other parts which have significant velocity offsets to the cluster systemic velocity. This chaotic 
velocity field supports our above scenario of a recent formation of the star pile and, indeed, also Salinas et al. suggests that the origin of the star pile is due to tidal stripping of some galaxy. However, their interpretation stress the importance of the central cluster potential and of the g3 galaxy as the most probable "central galaxy" of the cluster, not assigning any special role to the CBCG ("C" galaxy for Salinas et al.). Our interpretation stress the role of the CBCG and, indeed, looking at their Fig. 6 (upper panel, alternatively cf. their Table 4 and Fig. 5), the velocity of the $\mathrm{CBCG} / \mathrm{C}$ seems equally close or slightly closer to the star pile velocity than the velocity of g3. Finally, we note that Fig. 6 of Salinas et al. also reinforce our idea that the $\mathrm{cE} / \mathrm{g} 1$ is the remnant nucleus of a stripped galaxy since the star pile shows a positive velocity gradient from west - the region of the low-velocity $\mathrm{cE} / \mathrm{g} 1$ - to east towards the CBCG.

Acknowledgements. We are in debt with Federica Govoni for the VLA radio image and with Giuseppe Murante for useful discussions. We thank the referee for her/his remarks which allowed us to make the paper clearer and more complete. M.G. acknowledges financial support from ASI-INAF I/088/06/0 grant. This work has been also supported by the Programa Nacional de Astronomía y Astrofísica of the Spanish Ministry of Science and Innovation under grants AYA2010-21322-C03-02, AYA2007-67965-C03-01 and AYA2010-21887-C0404. This publication is based on observations made on the island of La Palma with the Italian Telescopio Nazionale Galileo (TNG) and the Isaac Newton Telescope (INT). The TNG is operated by the Fundación Galileo Galilei INAF (Istituto Nazionale di Astrofisica). The INT is operated by the Isaac Newton Group. Both telescopes are located in the Spanish Observatorio of the Roque de Los Muchachos of the Instituto de Astrofisica de Canarias. X-ray data are based on observations obtained with XMM-Newton, an ESA science mission with instruments and contributions directly funded by ESA Member States and NASA. This research has made use of the NASA/IPAC Extragalactic Database (NED), which is operated by the Jet Propulsion Laboratory, California Institute of Technology, under contract with the National Aeronautics and Space Administration.

\section{References}

Abell, G. O., Corwin, H. G. Jr., \& Olowin, R. P. 1989, ApJS, 70, 1 Anders, E., \& Grevesse, N. 1989, Geochimica et Cosmochimica Acta, 53, 197 Arnaud, K. A., 1996, in Astronomical Data Analysis Software and Systems V, ed. G. Jacoby, \& J. Barnes, ASP Conf. Ser., 101, 17

Ashman, K. M., Bird, C. M., \& Zepf, S. E. 1994, AJ, 108, 2348

Bacchi, M., Feretti, L., Giovannini, G., \& Govoni, F. 2003, A\&A, 400, 465

Bardelli, S., Zucca, E., Vettolani, G., et al. 1994, MNRAS, 267, 665

Barrena, R., Ramella, M., Boschin, W., et al. 2005, A\&A, 444, 685

Barrena, R., Boschin, W., Girardi, M., \& Spolaor, M. 2007a, A\&A, 467, 37

Barrena, R., Boschin, W., Girardi, M., \& Spolaor, M. 2007b, A\&A, 469, 861

Beers, T. C., \& Geller, M. J. 1983, ApJ, 274, 491

Beers, T. C., Geller, M. J., \& Huchra, J. P. 1982, ApJ, 257, 23

Beers, T. C., Flynn, K., \& Gebhardt, K. 1990, AJ, 100, 32

Beers, T. C., Forman, W., Huchra, J. P., Jones, C., \& Gebhardt, K. 1991, AJ, 102, 1581

Beers, T. C., Gebhardt, K., Huchra, J. P., et al. 1992, ApJ, 400, 410

Bekki, K., Couch, W. J., Drinkwater, M. J., \& Gregg, M. D. 2001, ApJ, 557, 39

Bell, E. F., Phleps, S., Somerville, R. S., et al. 2006, ApJ, 652, 270

Bertin, E., \& Arnouts, S. 1996, A\&AS, 117, 393

Binggelli, B. 1982, A\&A, 107, 338

Bird, C. M., \& Beers, T. C. 1993, AJ, 105, 1596

Biviano, A., \& Katgert, P. 2004, A\&A, 424, 779

Böhringer, H., Schuecker, P., Guzzo, L., et al. 2004, A\&A, 425, 367

Bonafede, A., Feretti, L., Giovannini, G., et al. 2009, A\&A, 503, 707

Boschin, W., Girardi, M., Barrena, R., et al. 2004, A\&A, 416, 839

Boschin, W., Girardi, M., Spolaor, M., \& Barrena, R. 2006, A\&A, 449, 461

Boschin, W., Barrena, R., Girardi, M., \& Spolaor, M. 2008, A\&A, 487, 33

Boschin, W., Barrena, R., \& Girardi, M. 2010, A\&A, 521, A78

Brunetti, G., Cassano, R., Dolag, K., \& Setti, G. 2009, A\&A, 507, 661

Bullock, J. S., Kolatt, T. S., Sigad, Y., et al. 2001, MNRAS, 321, 559

Buote, D. A. 2001, ApJ, 553, 15

Buote, D. A. 2002, in Merging Processes in Galaxy Clusters, Optcial Analysis

of Cluster Mergers, ed. L. Feretti, I. M. Gioia, \& G. Giovannini (The Netherlands: Kluwer Ac. Pub.)

Buote, D. A., \& Tsai, J. C. 1996, ApJ, 458, 27

Carlberg, R. G., Yee, H. K. C., \& Ellingson, E. 1997, ApJ, 478, 462
Cassano, R., \& Brunetti, G. 2005, MNRAS, 357, 1313

Cassano, R., Brunetti, G., \& Setti, G. 2006, MNRAS, 369, 1577

Cassano, R., Brunetti, G., Setti, G., Govoni, F., \& Dolag, K. 2007, MNRAS, 378, 1565

Cassano, R., Brunetti, G., Röttgering, H. J. A., \& Brüggen, M. 2010a, A\&A, 509, A68

Cassano, R., Ettori, S., Giacintucci, S., et al. 2010b, ApJ, 721, 82

Cavaliere, A., \& Fusco-Femiano, R. 1976, A\&A, 49, 137

Chilingarian, I., Cayatte, V., Chemin, L., et al. 2007, A\&A, 466, L21

Chilingarian, I., Cayatte, V., Revaz, Y., et al. 2009, Science, 326, 1379

Danese, L., De Zotti, C., \& di Tullio, G. 1980, A\&A, 82, 322

David, L. P., Slyz, A., Jones, C., et al. 1993, ApJ, 412, 479

den Hartog, R., \& Katgert, P. 1996, MNRAS, 279, 349

Dolag, K., \& Schindler, S. 2000, A\&A, 364, 491

Dolag, K., Bartelmann, M., Perrotta, F., et al. 2004, A\&A, 416, 853

Donnert, J., Dolag, K., Cassano, R., \& Brunetti, G. 2010, MNRAS, 407, 1565

Dressler, A., \& Shectman, S. A. 1988, AJ, 95, 985

Dubinsky, J. 1998, ApJ, 502, 141

Eckert, D., Molendi, S., \& Paltani, S. 2011, 526, 79

Ellingson, E., \& Yee, H. K. C. 1994, ApJS, 92, 33

Ensslin, T. A., \& Gopal-Krishna 2001, A\&A, 366, 26

Ensslin, T. A., Biermann, P. L., Klein, U., \& Kohle, S. 1998, A\&A, 332, 395

Ensslin, T. A., Pfrommer, C., Miniati, F., \& Subramanian, K. 2011, A\&A, 527, A99

Fadda, D., Girardi, M., Giuricin, G., Mardirossian, F., \& Mezzetti, M. 1996, ApJ, 473,670

Fasano, G., \& Franceschini, A. 1987, MNRAS, 225, 155

Feretti, L. 1999, MPE Report No. 271

Feretti, L. 2002, in The Universe at Low Radio Frequencies, Proc. IAU Symp. 199, held 30 Nov.-4 Dec. 1999, Pune, India, ed. A. Pramesh Rao, G. Swarup, \& Gopal-Krishna, 133

Feretti, L. 2005, X-Ray and Radio Connections, ed. L. O. Sjouwerman, \& K. K. Dyer. Published electronically by NRAO, http: //www . aoc.nrao.edu/ events/xraydio, held 3-6 February 2004 in Santa Fe, New Mexico, USA

Feretti, L., Gioia, I. M., \& Giovannini G. 2002, Merging Processes in Galaxy Clusters (The Netherlands: Kluwer Academic Publisher), Astrophysics and Space Science Library, 272

Ferrari, C., Maurogordato, S., Cappi, A., \& Benoist C. 2003, A\&A, 399, 813

Ferrari, C., Arnaud, M., Ettori, S., Maurogordato, S., \& Rho, J. 2006, A\&A, 446, 417

Ferrari, C., Govoni, F., Schindler, S., Bykov, A. M., \& Rephaeli, Y. 2008, Space Sci. Rev., 134, 93

Finoguenov, A., Sarazin, C. L., Nakazawa, K., Wik, D. R., \& Clarke, T. E. 2010, ApJ, 1143, 715

Fukugita, M., Shimasaku, K., \& Ichikawa, T. 1995, PASP, 107, 945

Giovannini, G., \& Feretti, L. 2002, in Merging Processes in Galaxy Clusters, Diffuse Radio Sources and Cluster Mergers, ed. L. Feretti, I. M. Gioia, \& G. Giovannini (The Netherlands: Kluwer Ac. Pub.)

Giovannini, G., Tordi, M., \& Feretti, L. 1999, New Astron., 4, 141

Giovannini, G., Bonafede, A., Feretti, L., et al. 2009, A\&A, 507, 1257

Girardi, M., \& Biviano, A. 2002, in Merging Processes in Galaxy Clusters, Optical Analysis of Cluster Mergers, ed. L. Feretti, I. M. Gioia, \& G. Giovannini (The Netherlands: Kluwer Ac. Pub.)

Girardi, M., \& Mezzetti, M. 2001, ApJ, 548, 79

Girardi, M., Fadda, D., Giuricin, G. et al. 1996, ApJ, 457, 61

Girardi, M., Escalera, E., Fadda, D., et al. 1997, ApJ, 482, 11

Girardi, M., Giuricin, G., Mardirossian, F., Mezzetti, M., \& Boschin, W. 1998, ApJ, 505, 74

Girardi, M., Barrena, R., \& Boschin, W. 2007, Contribution to Tracing Cosmic Evolution with Clusters of Galaxies: Six Years Later, conference - http:// www.si.inaf.it/sesto2007/contributions/Girardi.pdf

Girardi, M., Barrena, R., Boschin, W., \& Ellingson, E. 2008, A\&A, 491, 379

Girardi, M., Boschin, W., \& Barrena, R. 2010, A\&A, 517, A65

Goto, T., Sekiguchi, M., Nichol, R. C., et al. 2002, AJ, 123, 1807

Govoni, F., Murgia, M., Feretti, L., et al. 2005, A\&A, 430, L5

Gullixson, C. A. 1992, in Astronomical CCD Observing and Reduction techniques, ed. S. B. Howell, ASP Conf. Ser., 23, 130

Hashimoto, Y., Böhringer, H., Henry, J. P., Hasinger, G., \& Szokoly, G. 2007, A\&A, 467, 485

Henriksen, M. J., \& Tittley, E. R. 2002, ApJ, 577, 701

Henry, J. P., Briel, U. G., \& Nulsen, P. E. J. 1993, A\&A, 271, 413

Hoeft, M., Brüggen, M., \& Yepes, G. 2004, MNRAS, 347, 389

Kalberla, P. M. W., Burton, W. B., Hartmann, D., et al. 2005, A\&A, 440, 775 (LAB Map)

Kempner, J. C., Blanton, E. L., Clarke, T. E. et al. 2004, Proc. of the Conf., The Riddle of Cooling Flows in Galaxies and Clusters of Galaxies, ed. T. H. Reiprich, J. C. Kempner, \& N. Soker [arXiv:astro-ph/0310263], http://www.astro.virginia.edu/coolflow/ 
Kennicutt, R. C. 1992, ApJS, 79, 225

Keshet, U. 2011, MNRAS, submitted [arXiv: 1011.0729]

Kneib, J. P., Mellier, Y., Fort, B., \& Mathez, G. 1993, A\&A, 273, 367

Jones, C., \& Forman, W. 1992, in Clusters and Superclusters of Galaxies, ed. A.

C. Fabian (Dordrecht: Kluwer), 49

Landolt, A. U. 1992, AJ, 104, 340

Leccardi, A., \& Molendi, S. 2008, A\&A, 486, 359

Leccardi, A., Rossetti, M., \& Molendi, S. 2010, A\&A, 510, A82

Limber, D. N., \& Mathews, W. G. 1960, ApJ, 132, 286

Lin,Y.-T., \& Mohr, J. J. 2004, ApJ, 617, 879

Liu, F.S., Mao, S., Deng, Z. G., Xia, X. Y., \& Wen, Z. L. 2009, MNRAS, 396, 2003

Loeb, A., \& Mao, S. 1994, ApJ, 435, L109

Malumuth, E. M., Kriss, G. A., Dixon, W., Van Dyke, Ferguson, H. C., \& Ritchie, C. $1992, \mathrm{AJ}, 104,495$

Markevitch, M. 2010, Proceedings at 12th Marcel Grossman Meeting, Paris, July 2009 , updated with 2010 results [arXiv: 1010.3660 ]

Markevitch, M., Gonzalez, A. H., David, L., et al. 2002, ApJ, 567, L27

Markevitch, M., Govoni, F., Brunetti, G, \& Jerius, D. 2005, ApJ, 627, 733

Mastropietro, C., \& Burkert, A. 2008, MNRAS, 389, 967

Menci, N., \& Fusco-Femiano, R. 1996, ApJ, 472, 46

Merritt, D. 1984, ApJ, 276, 26

Million, E. T., \& Allen, S. W. 2009, MNRAS, 399, 1307

Murante, G., Giovalli, M., Gerhard, O., et al. 2007, 377, 2

Mushotzky, R. F., \& Scharf, C. A. 1997, ApJ, 482, L13

NAG Fortran Workstation Handbook 1986 (Downers Grove, IL: Numerical Algorithms Group)

Navarro, J. F., Frenk, C. S., \& White, S. D. M. 1997, ApJ, 490, 493

Niederste-Ostholt, M., Strauss, M. A., Dong, F., Koester, B. P., \& McKay, T. A. 2010, MNRAS, 405, 2023

Nieto, J.-L., \& Prugniel, P. 1987, A\&A, 186, 30

Okabe, N., \& Umetsu, K. 2008, PASJ, 60, 345

Owers, M. S., Randall, S. W., Nulsen, P. E., et al. 2011, ApJ, 728, 27
Pinkney, J., Roettiger, K., Burns, J.O., \& Bird, C.M. 1996, ApJS, 104, 1

Pisani, A. 1993, MNRAS, 265, 706

Pisani, A. 1996, MNRAS, 278, 697

Quintana, H., Carrasco, E. R., \& Reisenegger, A. 2000, AJ, 120, 511

Ramella, M., Boschin, W., Fadda, D., \& Nonino, M. 2001, A\&A, 368, 776

Ramella, M., Biviano, A., Pisani, A., et al. 2007, A\&A, 470, 39

Ricker, P. M., \& Sarazin, C. L. 2001, ApJ, 561, 621

Rines, K., Finn, R., \& Vikhlinin, A. 2007, ApJ, 665, 9

Roettiger, K., Burns, J. O., \& Loken, C. 1996, ApJ, 473, 651

Roettiger, K., Loken, C., \& Burns, J. O. 1997, ApJS, 109, 307

Roettiger, K., Burns, J. O., \& Stone, J. M. 1999, ApJ, 518, 603

Rossetti, M., Ghizzardi, S., Molendi, S., \& Finoguenov, A. 2007, A\&A, 463, 839

Rudick, C. S., Mihos, C., Frey, L. H., \& McBride, C. M. 2009, ApJ, 699, 1518

Salinas, R., Richtler, T., Romanowsky, A. J., West, M. J., \& Schuberth, Y. 2007, A\&A, 475, 507 (S07)

Salinas, R., Richtler, T., West, M. J., et al. 2011, A\&A, 528, A61

Sarazin, C. L. 2002, in Merging Processes in Galaxy Clusters, The Physics of Cluster Mergers, ed. L. Feretti, I. M. Gioia, \& G. Giovannini (The Netherlands: Kluwer Ac. Pub.)

Sarazin, C. L. 2004, J. Korean Astron. Soc., 37, 433

Sastry, G. M. 1968, PASP, 80, 252

Schlegel, D. J., Finkbeiner, D. P., \& Davis, M. 1998, ApJ, 500, 525

Schneider, D. P., Gunn, J. E., \& Hoessel, J. 1983, ApJ, 264, 337

Springel, V., \& Farrar, G. R. 2007, MNRAS, 380, 911

Struble, M. F. 1988, ApJ, 330, 25

Struble, M. F., \& Ftaclas, C. 1994, AJ, 108, 1

The, L. S., \& White, S. D. M. 1986, AJ, 92, 1248

Tonry, J., \& Davis, M. 1979, ApJ, 84, 1511

Tribble, P. C. 1993, MNRAS, 261, 57

van Dokkum, P. G. 2005, AJ, 130, 2647

Wainer, H., \& Schacht, S. 1978, Psychometrika, 43, 203

West, M. J., \& Bothun, G. D. 1990, ApJ, 350, 36

White, D. A., Jones, C., \& Forman, W. 1997, MNRAS, 292, 419 